TRANSACTIONS OF THE

AMERICAN MATHEMATICAL SOCIETY

Volume 361, Number 5, May 2009, Pages 2397-2429

S 0002-9947(08)04777-6

Article electronically published on December 16, 2008

\title{
POISSON GEOMETRY OF SL(3, C)-CHARACTER VARIETIES RELATIVE TO A SURFACE WITH BOUNDARY
}

\author{
SEAN LAWTON
}

\begin{abstract}
The $\mathrm{SL}(3, \mathbb{C})$-representation variety $\mathfrak{R}$ of a free group $\mathrm{F}_{r}$ arises naturally by considering surface group representations for a surface with boundary. There is an $\mathrm{SL}(3, \mathbb{C})$-action on the coordinate ring of $\mathfrak{R}$ by conjugation. The geometric points of the subring of invariants of this action is an affine variety $\mathfrak{X}$. The points of $\mathfrak{X}$ parametrize isomorphism classes of completely reducible representations. We show the coordinate ring $\mathbb{C}[\mathfrak{X}]$ is a complex Poisson algebra with respect to a presentation of $\mathrm{F}_{r}$ imposed by the surface. Lastly, we work out the bracket on all generators when the surface is a three-holed sphere or a one-holed torus.
\end{abstract}

\section{INTRODUCTION}

Surface group representations arise naturally in many contexts: moduli of geometric structures on surfaces G1, G2, knot and link invariants Si, spin networks $[\mathrm{LP}$, and moduli of flat principal bundles over a surface [GM, GHJW]. For surfaces with non-empty boundary and representations of their fundamental groups into subvarieties of $\mathrm{GL}_{m}$, the space of representations is an affine variety. The moduli of conjugacy classes of these representations (the categorical quotient) is likewise an affine variety. The algebraic structure of these varieties cannot alone distinguish the moduli for differing surfaces with the same Euler characteristic. However, there is additional structure making the moduli a Poisson variety. This additional structure distinguishes the moduli of surface group representations for surfaces with like Euler characteristic which are not homeomorphic. We demonstrate this for surfaces with $\chi=-1$. In general, if two non-homeomorphic surfaces have the same Euler characteristic, then they cannot have the same number of boundary components, and we will show that the boundary distinguishes the Poisson structure.

There has been much work in this area in recent years. A brief, albeit incomplete, history is in order. In the early 1980s, Goldman and Wolpert showed that there is a natural symplectic geometry on the space of surface group representations for a surface without boundary [G4, W]. In Goldman's paper he proves that the symplectic form is closed using the infinite dimensional space of all connections on a principal bundle over the surface. This prompted Karshon in 1992 [Ka to introduce an algebraic proof that the 2-form is closed. Then in 1997 the first proof that Goldman's 2-form extended to surfaces with boundary was published by Guruprasad, Huebschmann, Jeffrey, and Weinstein [GHJW], which was built on work that each had done previously. This 2 -form was also derived by Kim [Ki in

Received by the editors March 23, 2007.

2000 Mathematics Subject Classification. Primary 58D29; Secondary 14D20.

(C) 2008 American Mathematical Society Reverts to public domain 28 years from publication 
1999 in the context of convex projective structures. However, the moduli of surface group representations is not symplectic when the surface has non-empty boundary. It is foliated by symplectic subspaces and so is a Poisson variety. In 1986, following his previous work for closed surfaces, Goldman G5] described the Poisson bracket on the moduli in terms of intersections of cycles in homology. This work was later generalized by Chas and Sullivan in CS. Although it is reasonable to suppose Goldman's formula remains valid for surfaces with boundary, no proof exists in the literature. Moreover, the particular form of the Poisson structures is largely unexplored, as is the relationship between differing structures on the same variety. The only work to date exploring these structures directly is [G3, G6] in the case of $\mathrm{SL}(2, \mathbb{C})$ representations.

This paper is organized as follows. In Section 2 we introduce representation and character varieties (the moduli of representations) and review some of their properties. In Section 3 we show the moduli is symplectically foliated using the important work of [GHJW]. In Section 4 we recall Goldman's bracket formula and prove that his formulation extends to surfaces with boundary. Before computing the first known examples of $\mathrm{SL}(3, \mathbb{C})$ Poisson brackets, we review in Section 5 the structure of the character variety for Euler characteristic -1 surfaces, the only non-trivial case known (see [L). Then, in Sections 6 and 7 we come to the main theorems of this paper. We explicitly derive the Poisson structures for the threeholed sphere and the one-holed torus, and discuss symmetries in their formulations coming from the outer automorphisms of the surface group.

In particular, we show that with respect to certain symmetry operators coming from the aforementioned outer automorphisms the Poisson bi-vector field for the three-holed sphere is given by

$$
\mathfrak{a}_{4,-4} \frac{\partial}{\partial t_{(4)}} \wedge \frac{\partial}{\partial t_{(-4)}}+(1-\mathfrak{i})\left(\mathfrak{a}_{4,5} \frac{\partial}{\partial t_{(4)}} \wedge \frac{\partial}{\partial t_{(5)}}\right),
$$

whereas the bi-vector for the one-holed torus is given by

$$
\begin{aligned}
& \Sigma_{1}\left(\mathfrak{a}_{1,2} \frac{\partial}{\partial t_{(1)}} \wedge \frac{\partial}{\partial t_{(2)}}\right)+\Sigma_{2}\left(\mathfrak{a}_{3,4} \frac{\partial}{\partial t_{(3)}} \wedge \frac{\partial}{\partial t_{(4)}}\right) \\
& +\frac{1}{2} \Sigma_{1} \Sigma_{2}\left(\mathfrak{a}_{1,3} \frac{\partial}{\partial t_{(1)}} \wedge \frac{\partial}{\partial t_{(3)}}+\mathfrak{a}_{1,-3} \frac{\partial}{\partial t_{(1)}} \wedge \frac{\partial}{\partial t_{(-3)}}\right),
\end{aligned}
$$

despite the fact that both are identical as varieties. In each expression $\mathfrak{a}_{i, j}$ are polynomials explicitly derived in Sections [6] and 7.

\section{Representation AND CHARACTER VARIETIES OF A FREE GROUP}

2.1. Definition and properties. Let $\mathrm{F}_{r}$ be a rank $r$ free group generated by $\left\{\mathrm{x}_{1}, \ldots, \mathrm{x}_{r}\right\}$, and let $\mathfrak{G}$ be the linear algebraic group $\mathrm{SL}(m, \mathbb{C})$. The map

$$
\operatorname{Hom}\left(\mathrm{F}_{r}, \mathfrak{G}\right) \longrightarrow \mathfrak{G}^{\times r}
$$

defined by sending

$$
\rho \mapsto\left(\rho\left(\mathrm{x}_{1}\right), \rho\left(\mathrm{x}_{2}\right), \ldots, \rho\left(\mathrm{x}_{r}\right)\right)
$$

is a bijection. Since $\mathfrak{G}^{\times r}$ is the $r$-fold product of irreducible algebraic sets, $\mathfrak{G}^{\times r} \cong$ $\operatorname{Hom}\left(\mathrm{F}_{r}, \mathfrak{G}\right)$ is an affine variety. Moreover, since the product of smooth varieties over $\mathbb{C}$ is smooth, $\operatorname{Hom}\left(\mathrm{F}_{r}, \mathfrak{G}\right)$ is non-singular. As such we denote $\operatorname{Hom}\left(\mathrm{F}_{r}, \mathfrak{G}\right)$ by $\mathfrak{R}$ and refer to it as the $\mathfrak{G}$-representation variety of $\mathrm{F}_{r}$. 
Let $\mathbb{C}[\Re]$ be the coordinate ring of $\mathfrak{R}$. Our preceding remarks imply $\mathbb{C}[\Re] \cong$ $\mathbb{C}[\mathfrak{G}]^{\otimes r}$. For $1 \leq k \leq r$, define a generic matrix from the complex polynomial ring in $m^{2} r$ indeterminates by

$$
\mathbf{X}_{k}=\left(\begin{array}{cccc}
x_{11}^{k} & x_{12}^{k} & \cdots & x_{1 m}^{k} \\
x_{21}^{k} & x_{22}^{k} & \cdots & x_{2 m}^{k} \\
\vdots & \vdots & \ddots & \vdots \\
x_{m 1}^{k} & x_{m 2}^{k} & \cdots & x_{m m}^{k}
\end{array}\right)
$$

Let $\Delta$ be the ideal of relations defining $\mathfrak{R}$ in $\mathbb{C}\left[x_{i j}^{k} \mid 1 \leq i, j \leq m^{2}, 1 \leq k \leq r\right]$. Therefore, $\Delta=\left(\operatorname{det}\left(\mathbf{X}_{k}\right)-1 \mid 1 \leq k \leq r\right)$ and

$$
\mathbb{C}[\Re]=\mathbb{C}\left[x_{i j}^{k} \mid 1 \leq i, j \leq m^{2}, 1 \leq k \leq r\right] / \Delta .
$$

Let $\left(\mathbf{X}_{1}, \mathbf{X}_{2}, \ldots, \mathbf{X}_{r}\right)$ be an $r$-tuple of generic matrices. An element $f \in \mathbb{C}[\Re]$ is a function defined in terms of such $r$-tuples (unique up to an element in $\Delta$ ). There is a $\mathfrak{G}$-action on $\mathbb{C}[\mathfrak{R}]$ given by diagonal conjugation. Precisely, for $g \in \mathfrak{G}$

$$
g \cdot f\left(\mathbf{X}_{1}, \mathbf{X}_{2}, \ldots, \mathbf{X}_{r}\right)=f\left(g^{-1} \mathbf{X}_{1} g, \ldots, g^{-1} \mathbf{X}_{r} g\right) .
$$

We note that this is well defined since the generators of $\Delta$ are invariant under this action.

A linear algebraic group $\mathfrak{G}$ is called geometrically reductive if for any rational representation $\mathfrak{G} \rightarrow \mathrm{GL}_{m}$ and any non-zero invariant $v$ there exists an invariant homogeneous polynomial $f$ so $f(v) \neq 0$. It is a fact, proved by M. Nagata, that if $\mathfrak{G}$ is a geometrically reductive group acting rationally on an affine variety $\operatorname{Spec}_{\text {max }}(A)$, then $A^{\mathfrak{G}}$ is finitely generated. The "unitary trick" implies that $\mathrm{SL}(m, \mathbb{C})$ is geometrically reductive, and so the subring of invariants $\mathbb{C}[\Re]^{\mathfrak{G}}$ of the conjugation action defined above is a finitely generated $\mathbb{C}$-algebra (see $[\mathrm{D}, \mathrm{P} 1, \mathrm{R}]$ ).

The character variety

$$
\mathfrak{X}=\operatorname{Spec}_{\text {max }}\left(\mathbb{C}[\Re]^{\mathfrak{G}}\right)
$$

is the irreducible algebraic set whose coordinate ring is the ring of invariants. Therefore, $\mathbb{C}[\mathfrak{X}]$ includes all polynomial maps of the form $\operatorname{tr}\left(\mathbf{X}_{i_{1}} \mathbf{X}_{i_{2}} \cdots \mathbf{X}_{i_{k}}\right)$, where $1 \leq i_{j} \leq r$. However, it is a consequence of results of Procesi [P1] that all elements of the ring of invariants are polynomials in these functions. For $\mathfrak{G}=\operatorname{SL}(m, \mathbb{C})$, the maximal value of $k$ necessary in the expression $\operatorname{tr}\left(\mathbf{X}_{i_{1}} \mathbf{X}_{i_{2}} \cdots \mathbf{X}_{i_{k}}\right)$ is known to be between $m(m+1) / 2$ and $m^{2}$, and for $m=1,2,3,4$ it is known to be equal to the lower bound (see $[\mathrm{DF}]$ ).

For $r>1$, the Krull dimension of $\mathfrak{X}$ is $\left(m^{2}-1\right)(r-1)$ since generic elements have zero dimensional isotropy (see $[\mathrm{D}$, page 98 ).

There is a regular map $\mathfrak{R} \stackrel{\pi}{\rightarrow} \mathfrak{X}$ which factors through $\mathfrak{R} / \mathfrak{G}$ : let $\mathfrak{m}$ be a maximal ideal corresponding to a point in $\mathfrak{R}$; then the composite isomorphism $\mathbb{C} \rightarrow \mathbb{C}[\Re] \rightarrow$ $\mathbb{C}[\Re] / \mathfrak{m}$ implies that the composite map $\mathbb{C} \rightarrow \mathbb{C}[\mathfrak{R}]^{\mathfrak{G}} \rightarrow \mathbb{C}[\mathfrak{R}]^{\mathfrak{G}} /\left(\mathfrak{m} \cap \mathbb{C}[\Re]^{\mathfrak{G}}\right)$ is an isomorphism as well. Hence the contraction $\mathfrak{m} \cap \mathbb{C}[\mathfrak{R}]^{\mathfrak{G}}$ is maximal, and since for any $g \in \mathfrak{G},\left(g \mathfrak{m} g^{-1}\right) \cap \mathbb{C}[\mathfrak{R}]^{\mathfrak{G}}=\mathfrak{m} \cap \mathbb{C}[\mathfrak{R}]^{\mathfrak{G}}, \pi$ factors through $\mathfrak{R} / \mathfrak{G}$ (see $[\mathrm{E}$, page 38 ). Although $\mathfrak{R} / \mathfrak{G}$ is not generally an algebraic set, $\mathfrak{X}$ is the categorical quotient $\mathfrak{R} / / \mathfrak{G}$, and since $\mathfrak{G}$ is a (geometrically) reductive algebraic group, $\pi$ is surjective and maps closed $\mathfrak{G}$-orbits to points (see $[\mathrm{D}]$ ). 
2.2. Completely reducible representations. For every representation $\rho \in \mathfrak{R}$, $\mathbb{C}^{m}$ is an $\mathrm{F}_{r}$-module induced by $\rho$. A completely reducible representation is one that is a direct sum of irreducible subrepresentations. Such representations induce a semi-simple module structure on $\mathbb{C}^{m}$, and irreducible representations respectively result in simple modules. For any composition series of the $\mathrm{F}_{r}$-module associated to $\rho$,

$$
\mathbb{C}^{m}=V_{0} \supset V_{1} \supset \cdots \supset V_{l}=0,
$$

there is a semi-simple $\mathrm{F}_{r}$-module $W=\bigoplus V_{i} / V_{i+1}$. With respect to a chosen basis of $W$, there exists a completely reducible representation $\rho^{(s)}$. Its conjugacy class is independent of any basis, and moreover the Jordan-Hölder theorem implies that this class is also independent of the composition series.

We characterize these representations by their orbits when $m=3$ (the more general case is analogous) with the following proposition.

Proposition 1. Let $\mathfrak{G} \rho$ be the conjugation orbit of $\rho \in \mathfrak{R}$. Then $\mathfrak{G} \rho$ is closed if and only if $\rho$ is completely reducible.

Proof. If $\rho$ is not completely reducible $(l>1$ in (1) $)$, then it is reducible. It is either reducible by a dimension 1 or dimension 2 subspace. If it is reducible by a dimension 1 subspace, then there exists a basis so that for any $\mathrm{w} \in \mathrm{F}_{r}$ it has the form

$$
\left[\begin{array}{ccc}
a(\mathrm{w}) & b(\mathrm{w}) & c(\mathrm{w}) \\
0 & d(\mathrm{w}) & e(\mathrm{w}) \\
0 & f(\mathrm{w}) & g(\mathrm{w})
\end{array}\right] .
$$

In this form, conjugating by

$$
\left[\begin{array}{ccc}
1 & 0 & 0 \\
0 & 1 / n & 0 \\
0 & 0 & 1 / n
\end{array}\right]
$$

and taking the limit as $n \rightarrow \infty$ results in

$$
\left[\begin{array}{ccc}
a(\mathrm{w}) & 0 & 0 \\
0 & d(\mathrm{w}) & e(\mathrm{w}) \\
0 & f(\mathrm{w}) & g(\mathrm{w})
\end{array}\right] .
$$

This limiting representation is $\rho^{(s)}$ if it has two irreducible summands. Otherwise we may conjugate $\rho$ so $f(\mathrm{w})$ may be taken to be 0 . Then conjugating this form of $\rho$ by

$$
\left[\begin{array}{ccc}
1 & 0 & 0 \\
0 & 1 / n & 0 \\
0 & 0 & 1 / n^{2}
\end{array}\right]
$$

and taking the limit as $n \rightarrow \infty$ results in

$$
\left[\begin{array}{ccc}
a(\mathrm{w}) & 0 & 0 \\
0 & d(\mathrm{w}) & 0 \\
0 & 0 & g(\mathrm{w})
\end{array}\right]
$$

which is $\rho^{(s)}$ when it has three irreducible summands. Either way, we have a sequence, $\rho_{n} \in \mathfrak{G} \rho$, beginning at $\rho$ and limiting to $\rho^{(s)} \notin \mathfrak{G} \rho$. 
Now if $\rho$ is reducible by a dimension 2 subspace then there exists a basis so for any $\mathrm{w} \in \mathrm{F}_{r} \rho$ has the form:

$$
\left[\begin{array}{ccc}
a(\mathrm{w}) & b(\mathrm{w}) & c(\mathrm{w}) \\
d(\mathrm{w}) & e(\mathrm{w}) & f(\mathrm{w}) \\
0 & 0 & g(\mathrm{w})
\end{array}\right] .
$$

In this form, conjugating by

$$
\left[\begin{array}{ccc}
1 & 0 & 0 \\
0 & 1 & 0 \\
0 & 0 & 1 / n
\end{array}\right],
$$

and taking the limit as $n \rightarrow \infty$ results in

$$
\left[\begin{array}{ccc}
a(\mathrm{w}) & b(\mathrm{w}) & 0 \\
d(\mathrm{w}) & e(\mathrm{w}) & 0 \\
0 & 0 & g(\mathrm{w})
\end{array}\right] .
$$

This limiting representation is $\rho^{(s)}$ if it had two irreducible summands. Otherwise we may conjugate $\rho$ so $d($ w) may be taken to be 0 . Then conjugating this form of $\rho$ by

$$
\left[\begin{array}{ccc}
1 & 0 & 0 \\
0 & 1 / n & 0 \\
0 & 0 & 1 / n^{2}
\end{array}\right]
$$

and taking the limit as $n \rightarrow \infty$ results in

$$
\left[\begin{array}{ccc}
a(\mathrm{w}) & 0 & 0 \\
0 & e(\mathrm{w}) & 0 \\
0 & 0 & g(\mathrm{w})
\end{array}\right]
$$

which is $\rho^{(s)}$ when it has three irreducible summands. Again we have a sequence, $\rho_{n} \in \mathfrak{G} \rho$, beginning at $\rho$ and limiting to $\rho^{(s)} \notin \mathfrak{G} \rho$.

It follows that if $\mathfrak{G} \rho$ is closed, then $\rho$ is completely reducible.

For the converse, we first show that $\pi(\rho)=\pi(\psi)$ if and only if $\rho^{(s)}$ is conjugate to $\psi^{(s)}$. Indeed, suppose that $\pi(\rho)=\pi(\psi)$. Then their characteristic polynomials are equal: $\chi_{\rho}=\chi_{\psi}$. Thus $\chi_{\rho^{(s)}}=\chi_{\psi^{(s)}}$. However, semi-simple representations are determined by their characteristic polynomials (this is a non-trivial fact established in $[\mathrm{A}]$ ), so $\rho^{(s)}$ is conjugate to $\psi^{(s)}$. On the other hand, if for some basis $\rho^{(s)}=\psi^{(s)}$, then $\rho_{n} \rightarrow \rho^{(s)}=\psi^{(s)} \leftarrow \psi_{n}$. This in turn implies $\overline{\mathfrak{G} \rho} \cap \overline{\mathfrak{G} \psi}$ is not empty, and so $\pi(\rho)=\pi(\psi)$.

Now suppose $\rho$ has a non-closed orbit, and let $\psi$ be an element of $\overline{\mathfrak{G} \rho}-\mathfrak{G} \rho$. Then $\rho$ and $\psi$ are not conjugate. Without loss of generality, we can assume that $\mathfrak{G} \psi$ is closed since the dimension of each subsequent sub-orbit decreases. So $\psi=\psi^{(s)}$ and $\pi(\psi)=\pi(\rho)$. Hence, $\rho^{(s)}=\psi$, and so $\rho$ cannot be completely reducible; otherwise it would be conjugate to $\psi$, which it is not. In other words, if $\rho$ is completely reducible, then $\mathfrak{G} \rho$ is closed.

Let $\mathfrak{R}^{s s}$ be the subset of $\mathfrak{R}$ containing only completely reducible representations. Then we have just shown that $\mathfrak{R}^{s s}$ is the set of representations with closed orbits, $\mathfrak{R}^{s s} / \mathfrak{G}$ is in bijective correspondence (as sets) to $\mathfrak{X}$, and the following diagram 
commutes:

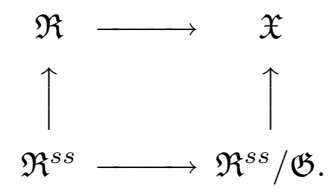

For a complete treatment of the above arguments see $[\mathrm{A}, \mathrm{P} 2$.

2.3. Simple representations. Let $\mathfrak{R}^{s} \subset \mathfrak{R}^{s s}$ be the set of irreducible representations, and let $\mathfrak{R}^{\text {reg }}$ be the regular points in $\mathfrak{R}$; that is, the representations that have closed orbits and have minimal dimensional isotropy. These points form an open dense subset of $\mathfrak{R}$ (see [D]).

Proposition 2. $\mathfrak{R}^{\text {reg }}=\mathfrak{R}^{s}$ if $\mathrm{F}_{r}$ has rank greater than 1 .

Proof. We have already seen that the irreducible representations have closed orbits, since they are completely reducible. So it remains to show that $\rho$ is irreducible if and only if its isotropy has minimal dimension. First, however, we address the case of $F_{1}$.

In this case, all representations have an invariant subspace, since the characteristic polynomial always has a root over $\mathbb{C}$. So there are no irreducible representations, and the semi-simple representations $\mathfrak{R}^{s s}$ are exactly the diagonalizable matrices since any matrix over $\mathbb{C}$ is conjugate to one in upper-triangular form. Moreover, the dimension of the isotropy of any such matrix is at least $m-1$, since any matrix commutes with itself. Also, the set of matrices with distinct eigenvalues is dense, and any diagonalizable matrix (the representations with closed orbits) has a repeated eigenvalue if and only if its isotropy has dimension strictly greater than $m-1$. So in this case, $\mathfrak{R}^{r e g}$ is the set of matrices with distinct eigenvalues, and $\mathfrak{R}^{s}=\emptyset$.

Otherwise, $\mathrm{F}_{r}$ has rank at least 2. If $\rho \in \mathfrak{R}^{s s}$ has an invariant subspace, it has non-zero dimensional isotropy since it fixes at least one line in $\mathbb{C}^{m}$. On the other hand, the representations that have at least two distinct generic matrices having no shared eigenspaces have isotropy equal to the center, which is generated by the $m^{\text {th }}$ roots of unity and so is zero dimensional. If a representation does not have this property, then it must be reducible. Hence, the minimal dimension of isotropy is zero, which is realized if and only if $\rho \in \mathfrak{R}^{s} \subset \mathfrak{R}^{s s}$. Thus when $\mathrm{F}_{r}$ has $r>1$, then $\mathfrak{R}^{\text {reg }}=\mathfrak{R}^{s}$.

In A, it is shown that $\mathfrak{R}^{s} / \mathfrak{G}$ is a non-singular variety. Moreover, in G2 it is shown that $\mathfrak{G}$ acts properly on $\mathfrak{R}^{s}$ (for $m=3$ only, but the argument generalizes), and although the action is not effective, the kernel is the center $\mathbb{Z}_{m}$. Thus the induced "infinitesimal" action on the tangent space is in fact effective, since the tangent map corresponding to the center is zero. Thus, if $\rho \in \mathfrak{R}^{s}$, the tangent space to an orbit, $T_{\rho}\left(\mathcal{O}_{\rho}\right)$, is isomorphic to $\mathfrak{g}$, the Lie algebra of $\mathfrak{G}$. Together with properness, this implies that $\mathfrak{R}^{s} \rightarrow \mathfrak{R} / / \mathfrak{G}$ is a local submersion which in turn implies $T_{\rho}(\mathfrak{R} / / \mathfrak{G}) \cong T_{\rho}(\mathfrak{R}) / T_{\rho}\left(\mathcal{O}_{\rho}\right)$ whenever $\rho$ is irreducible.

It is not always the case that the tangent space to the quotient is the quotient of tangent spaces. Just consider representations from the free group of rank 1 into $\operatorname{SL}(3, \mathbb{C})$. The ring of invariants is two dimensional, and the ring is generated by $\operatorname{tr}(\mathbf{X})$ and $\operatorname{tr}\left(\mathbf{X}^{-1}\right)$. So the ideal is zero and the ring is free. Consequently it is smooth, and the representation sending everything to the identity (having 
maximal isotropy) is a non-singular point. This illustrates that there can be smooth points in the quotient that have positive dimensional isotropy. At these points, $T_{\rho}(\mathfrak{R} / / \mathfrak{G}) \nsucceq T_{\rho}(\mathfrak{R}) / T_{\rho}\left(\mathcal{O}_{\rho}\right)$, seen by simply comparing dimensions.

Comment 3. In the sense of Geometric Invariant Theory (GIT), the semistable points of the $\mathfrak{G}$ action are the entire space $\mathfrak{R}$, since for affine reductive $\mathfrak{G}$ varieties all points are semistable in general (there are no points for which all invariants vanish). So the set of semisimple representations is not equal to the set of semistable points. The semisimple representations are sometimes referred to as polystable in GIT. However, the stable points are in fact the simple representations. We make this comment since the $s$ in the notation $\mathfrak{R}^{s}$ can be taken to mean either stable or simple, but the $s s$ in the notation $\mathfrak{R}^{s s}$ means only semisimple (not semistable).

\section{Symplectid FOLIATIONS ON CHARACTER VARIETIES}

3.1. The boundary map and generic leaves. Let $\Sigma_{n, g}$ be a compact, connected, smooth, orientable surface of genus $g$ with $n>0$ disjoint open disks removed, and let $*$ be a generic point in $\Sigma_{n, g}$. Its fundamental group has the following presentation:

$\pi_{1}\left(\Sigma_{n, g}, *\right)=\left\{\mathrm{x}_{1}, \mathrm{y}_{1}, \ldots, \mathrm{x}_{g}, \mathrm{y}_{g}, \mathrm{~b}_{1}, \ldots, \mathrm{b}_{n} \mid \mathrm{x}_{1} \mathrm{y}_{1} \mathrm{x}_{1}^{-1} \mathrm{y}_{1}^{-1} \cdots \mathrm{x}_{g} \mathrm{y}_{g} \mathrm{x}_{g}^{-1} \mathrm{y}_{g}^{-1} \mathrm{~b}_{1} \cdots \mathrm{b}_{n}=1\right\}$, which is free of rank $r=2 g+n-1$, and so its Euler characteristic is $\chi\left(\Sigma_{n, g}\right)=$ $1-r+0=2-2 g-n$. We will refer to such a surface as $n$-holed. So the following is a two-holed genus two surface:

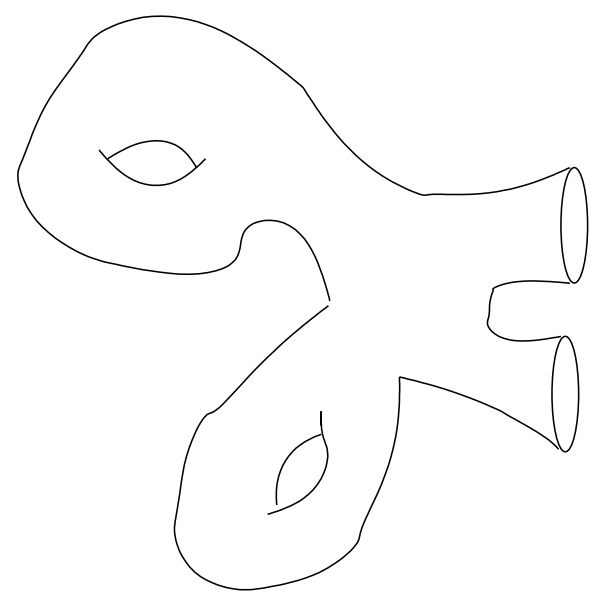

FiguRE 1. $\Sigma_{2,2}$

If we assume $r>0$, then $\chi \leq 0$. In particular, $r=1$ if and only if $\chi=0$, and in this case $\Sigma_{2,0}$ is homeomorphic to an annulus. Otherwise, $\chi<0$. The rank $r$ is 2 if and only if $\chi=-1$, in which case the surface is either $\Sigma_{3,0}$ or $\Sigma_{1,1}$; that is, the three-holed sphere (or trinion or pair-of-pants), or the one-holed torus, respectively.

Let $\mathfrak{G}=\mathrm{SL}(m, \mathbb{C})$ and let $\mathbb{I}$ be the $m \times m$ identity matrix. The coordinate ring of $\mathfrak{G} / / \mathfrak{G}$ is freely generated by the $m-1$ coefficients of the characteristic polynomial

$$
\operatorname{det}(\lambda \mathbb{I}-\mathbf{X})=\lambda^{m}-c_{1}(\mathbf{X}) \lambda^{m-1}+\cdots \pm c_{m-1}(\mathbf{X}) \lambda \mp 1
$$


in $\mathbb{C}[\mathfrak{G}]$. Consequently, $\mathfrak{G} / / \mathfrak{G}=\mathbb{C}^{m-1}$, which we parametrize by coordinates $\left(\tau_{(1)}, \ldots, \tau_{(m-1)}\right)$. We note that $c_{1}(\mathbf{X})=\operatorname{tr}(\mathbf{X})$ and $c_{m-1}(\mathbf{X})=\operatorname{tr}\left(\mathbf{X}^{-1}\right)$ in general.

We make the following definition in these terms.

Definition 4. The $i^{\text {th }}$ boundary map

$$
\mathfrak{b}_{i}: \mathfrak{X}=\mathfrak{R} / / \mathfrak{G}=\operatorname{Hom}\left(\pi_{1}\left(\Sigma_{n, g}, *\right), \mathfrak{G}\right) / / \mathfrak{G} \longrightarrow \mathfrak{G} / / \mathfrak{G}
$$

is the mapping $[\rho] \mapsto\left[\rho_{\left.\right|_{\mathrm{b}_{i}}}\right]=\left(\tau_{(1)}^{i}, \ldots, \tau_{(m-1)}^{i}\right)$ which sends the extended class of a representation to the class corresponding to the restriction of $\rho$ to the boundary $\mathrm{b}_{i}$.

This is well defined since there exists a representative $\rho$ of $[\rho]$ whose orbit is of maximal dimension (necessarily unique) and for any $g \in \mathfrak{G}$ the orbit of $\mathbf{B}_{i}=\rho\left(\mathbf{b}_{i}\right)$ and $g \rho\left(\mathbf{b}_{i}\right) g^{-1}$ are identical. However, the map $\mathfrak{b}_{i}$ is invariant and polynomial, and any other representative from $[\rho]$ is in the closure of the orbit $\mathfrak{G} \rho$. Hence it is well defined for any representative of $[\rho]$.

Subsequently, we make the following further definition.

Definition 5. The boundary map is the mapping $\mathfrak{b}=\left(\mathfrak{b}_{1}, \ldots, \mathfrak{b}_{n}\right): \mathfrak{X}=\mathfrak{G}^{\times r} / / \mathfrak{G} \longrightarrow$ $(\mathfrak{G} / / \mathfrak{G})^{\times n}$.

The boundary map $\mathfrak{b}$ depends on the surface, in particular the presentation of its fundamental group. We refer to it as a peripheral structure and to the pair $(\mathfrak{X}, \mathfrak{b})$ as the relative character variety.

Let

$\mathfrak{F}=\bigcap_{i=1}^{n} \mathfrak{b}_{i}^{-1}\left(\tau_{(1)}^{i}, \ldots, \tau_{(m-1)}^{i}\right)=\left\{[\rho] \mid \mathfrak{b}([\rho])=\left(\left(\tau_{(1)}^{1}, \ldots, \tau_{(m-1)}^{1}\right), \ldots,\left(\tau_{(1)}^{n}, \ldots, \tau_{(m-1)}^{n}\right)\right)\right\}$.

Each $\mathfrak{F}$ is cut out of $\mathfrak{X}$ by the equations $c_{k}\left(\mathbf{B}_{i}\right)=\tau_{(k)}^{i}$ for $1 \leq k \leq m-1$ and $1 \leq i \leq n$, and so is an algebraic set. Moreover, they partition $\mathfrak{X}$ since every representation has well-defined boundary values.

The dimension of $\mathfrak{X}$ is

$$
\left(m^{2}-1\right)(r-1)=2\left(m^{2}-1\right)(g-1)+\left(m^{2}-1\right) n,
$$

and imposing boundary values provide $(m-1) n$ relations (not necessarily independent) in $\mathbb{C}[\mathfrak{X}]$. Therefore, the dimension of any $\mathfrak{F}$ is greater than or equal to $2\left(m^{2}-1\right)(g-1)+m(m-1) n$.

We now prove the following proposition which we need to show that this partitioning of $\mathfrak{X}$ is in fact a foliation of its smooth stratum.

Proposition 6. If $g \geq 1$, then $\mathfrak{b}$ is surjective. In all other cases, $\mathfrak{b}$ is dominant; that is, $\overline{\mathfrak{b}(\mathfrak{X})}=\mathbb{C}^{n(m-1)}$.

Proof. We first suppose $g \geq 1$. The mapping $\mathrm{GL}(m, \mathbb{C}) \times \mathrm{GL}(m, \mathbb{C}) \rightarrow \operatorname{SL}(m, \mathbb{C})$ given by $(\mathbf{A}, \mathbf{B}) \mapsto[\mathbf{A}, \mathbf{B}]=\mathbf{A B} \mathbf{A}^{-1} \mathbf{B}^{-1}$ is surjective (see $[\mathrm{Fo}]$ ). This easily implies that the mapping $\kappa_{1}: \mathfrak{G} \times \mathfrak{G} \rightarrow \mathfrak{G}$ is surjective by simply choosing $m^{\text {th }}$ roots of the determinants of $\mathbf{A}$ and $\mathbf{B}$. In fact, we have that the mapping $\kappa_{g}: \mathfrak{G}^{\times 2 g} \rightarrow \mathfrak{G}$ given by $\left(\mathbf{A}_{1}, \mathbf{B}_{1}, \ldots, \mathbf{A}_{g}, \mathbf{B}_{g}\right) \mapsto\left[\mathbf{A}_{1}, \mathbf{B}_{1}\right] \cdots\left[\mathbf{A}_{g}, \mathbf{B}_{g}\right]$ is also surjective simply by letting $\mathbf{A}_{2}=\mathbf{B}_{2}=\cdots \mathbf{A}_{g}=\mathbf{B}_{g}=\mathbb{I}$ and picking a value for $\kappa_{1}$.

Now take any point $p=\left(\left(\tau_{(1)}^{1}, \ldots, \tau_{(m-1)}^{1}\right), \ldots,\left(\tau_{(1)}^{n}, \ldots, \tau_{(m-1)}^{n}\right)\right)$ in $\mathbb{C}^{n(m-1)}$. We wish to find $[\rho] \in \mathfrak{X}$ so $\mathfrak{b}([\rho])=p$. Let $r=2 g+n-1$, and let $\mathfrak{R}=\mathfrak{G}^{\times r}$ be the subset of $\mathfrak{R} \times \mathfrak{G}$ parametrized by

$$
\left(\mathbf{X}_{1}, \mathbf{Y}_{1}, \ldots, \mathbf{X}_{g}, \mathbf{Y}_{g}, \mathbf{B}_{1}, \ldots, \mathbf{B}_{n-1},\left(\Pi_{i=1}^{g}\left[\mathbf{X}_{i}, \mathbf{Y}_{i}\right] \Pi_{j=1}^{n-1} \mathbf{B}_{j}\right)^{-1}\right)
$$


For all $1 \leq k \leq n$ and every tuple $\left(\tau_{(1)}^{k}, \ldots, \tau_{(m-1)}^{k}\right)$ there exists a matrix $\mathbf{B}_{k}$ so $c_{j}\left(\mathbf{B}_{k}\right)=\tau_{j}^{k}$ for $1 \leq j \leq m-1$ since there exists a global section to $\mathfrak{G} \rightarrow \mathfrak{G} / / \mathfrak{G}$ given by the rational canonical form of a matrix. Now let $\mathbf{Z}=\left(\mathbf{B}_{1} \cdots \mathbf{B}_{n}\right)^{-1}$. Then there exists $q=\left(\mathbf{X}_{1}, \mathbf{Y}_{1}, \ldots, \mathbf{X}_{g}, \mathbf{Y}_{g}\right)$ so $\kappa_{g}(q)=\mathbf{Z}$. Then it follows that $\rho:=\left(q, \mathbf{B}_{1}, \ldots, \mathbf{B}_{n}\right)$ is in $\mathfrak{R} \subset \mathfrak{R} \times \mathfrak{G}$. Letting $[\rho]=\pi(\rho) \in \mathfrak{X}$, we have that $\mathfrak{b}([\rho])=p$.

Now if $g=0$ the mapping may not be surjective (this is related to the DeligneSimpson problem). However, it is dominant; that is, its image is dense.

Let $\mathcal{O}$ be the subset of $\mathfrak{G}$ defined by the property that all eigenvalues are distinct and no proper subset of the eigenvalues multiplies to 1. Foth [Fo] calls the latter of these two conditions property $P$, and Kostov $[\mathrm{Ko}$. says such matrices have generic eigenvalues. Both conditions define Zariski open sets and so the intersection of these two sets is likewise Zariski open. Hence, the $m-1$ coefficients of the characteristic polynomial, $\left\{c_{1}, \ldots, c_{m-1}\right\}$, are independent on this open dense set since they are polynomial and independent globally. Consequently, the image of the $n(m-1)$ tuple $\left(\left(c_{1}, \ldots, c_{m-1}\right), \ldots,\left(c_{1}, \ldots, c_{m-1}\right)\right)$ from $\mathcal{O}^{n}$ to $\mathbb{C}^{n(m-1)}$ is dense. Call the image $c(\mathcal{O})$. Take any point $p \in c(\mathcal{O})$. We then have $n$ conjugacy classes in $\mathfrak{G}: C_{1}, \ldots, C_{n}$, where each has representatives with distinct and generic eigenvalues. It is shown in $[\mathrm{Ko}]$ that the set

$$
\mathcal{W}\left(C_{1}, \ldots, C_{n}\right):=\left\{\left(M_{1}, \ldots, M_{n}\right) \mid M_{i} \in C_{i}, M_{1} \cdots M_{n}=\mathbb{I}\right\}
$$

is a smooth connected variety of positive dimension $\sum \operatorname{dim} C_{i}-\left(m^{2}-1\right)$. Now take any such tuple of $n$ matrices; then it defines a representation $\rho: \pi_{1}\left(\Sigma_{0, n}, *\right) \rightarrow \mathfrak{G}$ and so $\mathfrak{b}([\rho])=p$. Hence $c(\mathcal{O}) \subset \operatorname{Image}(\mathfrak{b})$. Since $\overline{c(\mathcal{O})}=\mathbb{C}^{n(m-1)}, \mathfrak{b}$ is dominant.

Consequently, we have the following corollary (see [Ha], page 271).

Corollary 7. There exists an open dense subset (Zariski open) $\mathfrak{X}^{\mathfrak{b}} \subset \mathfrak{X}$ where $\mathfrak{b}$ is a submersion.

Let $\mathcal{X}$ be the complement of the singular locus (a proper sub-variety) in $\mathfrak{X}$, so $\mathcal{X}$ is a non-singular complex manifold that is dense in $\mathfrak{X}$. The construction of $\mathfrak{X}^{\mathfrak{b}}$ provides that $\mathfrak{X}^{\mathfrak{b}} \subset \mathcal{X}$. Thus the implicit function theorem (see [Wa] ) on the smooth complex manifold $\mathfrak{X}^{\mathfrak{b}}$ gives that $\mathcal{F}:=\mathfrak{F} \cap \mathfrak{X}^{\mathfrak{b}}$ is a non-singular complex submanifold of dimension $\left(m^{2}-1\right)(r-1)-(m-1) n=2\left(m^{2}-1\right)(g-1)+m(m-1) n$. In particular, these submanifolds are of even complex dimension.

Since $\mathfrak{b}$ is a submersion here, the union of these leaves, $\mathcal{F}$, foliate $\mathfrak{X}^{\mathfrak{b}}$. We will show that these leaves are complex symplectic submanifolds which extend to make $\mathcal{X}$ a complex Poisson manifold. Moreover, the Poisson structure extends continuously over singularities in $\mathfrak{X}$. With respect to this structure, we will refer to the relative character variety $(\mathfrak{X}, \mathfrak{b})$ as a Poisson variety; in particular, an affine variety whose coordinate ring is a Poisson algebra. We will often drop the fact that we are working over $\mathbb{C}$, but whenever we are discussing a symplectic structure we are referring to a closed $(2,0)$-form.

3.2. Tangents, cocycles, and coboundaries. Let $\mathrm{F}_{r}$ be a free group of rank $r$, and let $\mathfrak{g}$ be the Lie algebra of $\mathfrak{G}$ identified with its right invariant vector fields. 
For $\rho \in \mathfrak{R}, \mathfrak{g}$ is a $\mathrm{F}_{r}$-module, $\mathfrak{g}_{\mathrm{Ad}_{\rho}}$, given by

$$
\mathrm{F}_{r} \stackrel{\rho}{\longrightarrow} \mathfrak{G} \stackrel{\mathrm{Ad}}{\longrightarrow} \operatorname{Aut}(\mathfrak{g}),
$$

where $\operatorname{Ad}(\rho(\mathrm{w}))(X)=\rho(\mathrm{w}) X \rho(\mathrm{w})^{-1}$ is the adjoint representation.

Define $C^{0}\left(\mathrm{~F}_{r} ; \mathfrak{g}_{\mathrm{Ad}_{\rho}}\right)=\mathfrak{g}$ and $C^{n}\left(\mathrm{~F}_{r} ; \mathfrak{g}_{\mathrm{Ad}_{\rho}}\right)=\left\{\mathrm{F}_{r}^{\times n} \rightarrow \mathfrak{g}\right\}$, the vector space of functions $\mathrm{F}_{r}^{\times n} \rightarrow \mathfrak{g}$. Now define $\delta_{n}: C^{n}\left(\mathrm{~F}_{r} ; \mathfrak{g}_{\mathrm{Ad}_{\rho}}\right) \rightarrow C^{n+1}\left(\mathrm{~F}_{r} ; \mathfrak{g}_{\mathrm{Ad}_{\rho}}\right)$ by

$$
\begin{aligned}
\delta_{n} f\left(\mathrm{w}_{1}, \mathrm{w}_{2}, \ldots, \mathrm{w}_{n+1}\right)= & \operatorname{Ad}_{\rho}\left(\mathrm{w}_{1}\right) f\left(\mathrm{w}_{2}, \ldots, \mathrm{w}_{n+1}\right)+\sum_{i=1}^{n}(-1)^{i} f\left(\mathrm{w}_{1}, \ldots, \mathrm{w}_{i} \mathrm{w}_{i+1}, \ldots, \mathrm{w}_{n+1}\right) \\
& +(-1)^{n+1} f\left(\mathrm{w}_{1}, \ldots, \mathrm{w}_{n}\right) .
\end{aligned}
$$

One may verify that $\delta_{n+1} \circ \delta_{n}=0$, and so $\left(C^{*}\left(\mathrm{~F}_{r} ; \mathfrak{g}_{\mathrm{Ad}_{\rho}}\right), \delta_{*}\right)$ is a cochain complex with coboundary operator $\delta_{*}$.

Let $\mathfrak{G}_{r}^{\mathrm{F}_{r} n}$ be the set of functions $\mathrm{F}_{r}^{\times n} \rightarrow \mathfrak{G}$. Let $f_{t}$ be a curve in $\mathfrak{G}_{r}^{\mathrm{F}_{r}}$, and for $\left(\mathrm{w}_{1}, \ldots, \mathrm{w}_{n}\right) \in \mathrm{F}_{r}^{\times n}$ let $\epsilon_{\left(\mathrm{w}_{1}, \ldots, \mathrm{w}_{n}\right)}\left(f_{t}\right)=f_{t}\left(\mathrm{w}_{1}, \ldots, \mathrm{w}_{n}\right)$ be the evaluation function. Then for each evaluation, we have a curve in $\mathfrak{G}$, and so we say $f_{t}$ is smooth if and only if it is smooth at all evaluations (see $\mathrm{Ka}$ ). We define the tangent space at a function $f$ to be the vector space of tangents to smooth curves $f_{t}$ where $f_{0}=f$. In other words,

given by

$$
T_{f}\left(\mathfrak{G}^{\mathrm{F}_{r}^{\times n}}\right) \cong \mathfrak{g}^{\mathrm{F}_{r}^{\times n}}=C^{n}\left(\mathrm{~F}_{r} ; \mathfrak{g}_{\mathrm{Ad}_{\rho}}\right)
$$

$$
u_{\left(\mathrm{w}_{1}, \ldots, \mathrm{w}_{n}\right)}(f)=\left.\frac{\mathrm{d}}{\mathrm{d} t}\right|_{t=0} \epsilon_{\left(\mathrm{w}_{1}, \ldots, \mathrm{w}_{n}\right)}\left(f_{t}\right)=\left.\frac{\mathrm{d}}{\mathrm{d} t}\right|_{t=0} \exp \left(t \alpha_{\left(\mathrm{w}_{1}, \ldots, \mathrm{w}_{n}\right)}\right) \epsilon_{\left(\mathrm{w}_{1}, \ldots, \mathrm{w}_{n}\right)} f,
$$

where $\alpha \in \mathfrak{g}_{r}^{\mathrm{F}_{r}^{\times n}}$. Since a function is determined by its evaluations, we consider right invariant vector fields defined along these coordinates which give paths with the requisite properties.

Let $I$ be a finite subset of $\mathrm{F}_{r}$, and let $\mathbb{C}\left[\mathfrak{G}^{I}\right]$ be the coordinate ring of $\mathfrak{G}^{I}$. The set of such $I$ 's is partially ordered by set inclusion, and so $\mathbb{C}\left[\mathfrak{G}^{J}\right] \hookrightarrow \mathbb{C}\left[\mathfrak{G}^{I}\right]$ for $J \subset I$. We then define $\mathbb{C}\left[\mathfrak{G}^{\mathrm{F}_{r}}\right]=\lim _{\longrightarrow}\left[\mathfrak{G}^{I}\right]$. With this said, we note that $\mathfrak{R}$ is the subspace of $\mathfrak{G}^{\mathrm{F}_{r}}$ that is cut out by the functions ob $\mathrm{x}, \mathrm{y}(f)=f(\mathrm{x}) f(\mathrm{y}) f(\mathrm{xy})^{-1}$. In other words, letting $\mathbb{I}$ be the $m \times m$ identity matrix,

$$
\mathbb{C}[\Re]=\mathbb{C}\left[\mathfrak{G}^{\mathrm{F}_{r}}\right] /\left(\mathrm{ob}_{\mathrm{x}, \mathrm{y}}-\mathbb{I} \mid \mathrm{x}, \mathrm{y} \in \mathrm{F}_{r}\right)
$$

and $\mathfrak{R}=\operatorname{Spec}_{\max }(\mathbb{C}[\mathfrak{R}])$.

The following proposition is a classical fact which we prove here to keep the discussion self-contained.

Proposition 8. If $\rho \in \mathfrak{R}^{\text {reg }}=\mathfrak{R}^{s} \subset \mathfrak{R}^{s s}$, and $[\rho]=\pi(\rho) \in \mathfrak{X}$, then

$$
T_{[\rho]}(\mathfrak{X}) \cong T_{\rho}(\mathfrak{R}) / T_{\rho}\left(\mathcal{O}_{\rho}\right)=Z^{1}\left(\mathrm{~F}_{r} ; \mathfrak{g}_{\mathrm{Ad}_{\rho}}\right) / B^{1}\left(\mathrm{~F}_{r} ; \mathfrak{g}_{\mathrm{Ad}_{\rho}}\right)=H^{1}\left(\mathrm{~F}_{r} ; \mathfrak{g}_{\mathrm{Ad}_{\rho}}\right) .
$$

Proof. In the terms outlined above, $T_{f}(\mathfrak{R})=\left\{u \in T_{f}\left(\mathfrak{G}^{\mathrm{F}_{r}}\right) \mid u\left(f(\mathrm{x}) f(\mathrm{y}) f(\mathrm{xy})^{-1}\right)=\right.$ $0\}$, and so

$$
\begin{aligned}
0= & u\left(f(\mathrm{x}) f(\mathrm{y}) f(\mathrm{xy})^{-1}\right) \\
= & u(f(\mathrm{x})) f(\mathrm{y}) f(\mathrm{xy})^{-1}+f(\mathrm{x}) u(f(\mathrm{y})) f(\mathrm{xy})^{-1} \\
& -f(\mathrm{x}) f(\mathrm{y}) f(\mathrm{xy})^{-1} u(f(\mathrm{xy})) f(\mathrm{xy})^{-1}
\end{aligned}
$$

which implies $u_{\mathrm{xy}}=u_{\mathrm{x}}+\operatorname{Ad}_{f}(\mathrm{x}) u_{\mathrm{y}}$. 
However, this is exactly the condition for $\delta_{1}(f)=0$, and so

$$
T_{f}(\mathfrak{R})=\operatorname{Ker}\left(\delta_{1}\right)=Z^{1}\left(\mathrm{~F}_{r} ; \mathfrak{g}_{\mathrm{Ad}_{f}}\right) .
$$

On the other hand, consider a smooth path contained in the orbit $\mathcal{O}_{f} \subset \mathfrak{R}$ :

$$
f_{t}(\mathrm{x})=\exp \left(t u_{\mathrm{x}}\right) f(\mathrm{x})=\exp \left(-t u_{0}\right) f(\mathrm{x}) \exp \left(t u_{0}\right),
$$

for some $u_{0} \in \mathfrak{g}=C^{0}\left(\mathrm{~F}_{r} ; \mathfrak{g}_{\mathrm{Ad}_{\rho}}\right)$. Then

$$
u_{\mathrm{x}} f(\mathrm{x})=\left.\frac{\mathrm{d}}{\mathrm{d} t}\right|_{t=0} f_{t}(\mathrm{x})=-u_{0} f(\mathrm{x})+f(\mathrm{x}) u_{0}
$$

which implies $u_{\mathrm{x}}=\operatorname{Ad}_{f}(\mathrm{x}) u_{0}-u_{0}$. However this is exactly the condition $\delta_{0}\left(u_{0}\right)=u$, so

$$
T_{f}\left(\mathcal{O}_{f}\right)=\operatorname{Image}\left(\delta_{0}\right)=B^{1}\left(\mathrm{~F}_{r} ; \mathfrak{g}_{\mathrm{Ad}_{f}}\right) .
$$

Therefore, if $\rho \in \mathfrak{R}^{\text {reg }}=\mathfrak{R}^{s} \subset \mathfrak{R}^{s s}$, and $[\rho]=\pi(\rho) \in \mathfrak{X}$, then

$$
T_{[\rho]}(\mathfrak{X}) \cong T_{\rho}(\mathfrak{R}) / T_{\rho}\left(\mathcal{O}_{\rho}\right)=Z^{1}\left(\mathrm{~F}_{r} ; \mathfrak{g}_{\mathrm{Ad}_{\rho}}\right) / B^{1}\left(\mathrm{~F}_{r} ; \mathfrak{g}_{\mathrm{Ad}_{\rho}}\right)=H^{1}\left(\mathrm{~F}_{r} ; \mathfrak{g}_{\mathrm{Ad}_{\rho}}\right),
$$

as was to be shown.

3.3. Homology and the fundamental cycle. Let $\mathbb{Z F}_{r}$ be the integral group ring of $\mathrm{F}_{r}$, and let $\epsilon: \mathbb{Z F}_{r} \rightarrow \mathbb{Z}$ be the augmentation map defined by $\sum n_{\mathrm{w}} \mathrm{W} \mapsto \sum n_{\mathrm{w}}$. Define $\mathbb{Z}$-modules $C_{0}\left(\mathrm{~F}_{r}\right)=\mathbb{Z}$ and $C_{n}\left(\mathrm{~F}_{r}\right)=\mathbb{Z F}_{r}^{\times n}$, and let

$$
\partial_{n+1}: C_{n+1}\left(\mathrm{~F}_{r}\right) \longrightarrow C_{n}\left(\mathrm{~F}_{r}\right)
$$

be defined by

$$
\begin{aligned}
\partial_{n+1}\left(\mathrm{w}_{1}, \mathrm{w}_{2}, \ldots, \mathrm{w}_{n+1}\right)= & \epsilon\left(\mathrm{w}_{1}\right)\left(\mathrm{w}_{2}, \ldots, \mathrm{w}_{n+1}\right)+\sum_{i=1}^{n}(-1)^{i}\left(\mathrm{w}_{1}, \ldots, \mathrm{w}_{i} \mathrm{w}_{i+1}, \ldots, \mathrm{w}_{n+1}\right) \\
& +(-1)^{n+1}\left(\mathrm{w}_{1}, \ldots, \mathrm{w}_{n}\right) \epsilon\left(\mathrm{w}_{n+1}\right) .
\end{aligned}
$$

One can show $\partial_{n} \circ \partial_{n+1}=0$, and so $\left(C_{*}\left(\mathrm{~F}_{r}\right), \partial_{*}\right)$ is a chain complex with boundary $\partial_{*}$.

In $\left[\mathrm{F}\right.$ it is shown that the derivations on $\mathbb{Z} \mathrm{F}_{r}$,

$$
\operatorname{Der}\left(\mathrm{F}_{r}\right)=\left\{D: \mathbb{Z F}_{r} \rightarrow \mathbb{Z F}_{r} \mid D(\mathrm{xy})=D(\mathrm{x}) \epsilon(\mathrm{y})+\mathrm{x} D(\mathrm{y})\right\},
$$

are freely generated by the derivations $\frac{\partial}{\partial \mathrm{x}_{i}}\left(\mathrm{x}_{j}\right)=\delta_{i j}$. These derivations and their generators are called the Fox derivatives.

Recall that the peripheral structure on $\mathfrak{X}$ is given by the presentation

$$
\mathrm{F}_{r}=\pi_{1}\left(\Sigma_{n, g}, *\right)=\left\{\mathrm{x}_{1}, \mathrm{y}_{1}, \ldots, \mathrm{x}_{g}, \mathrm{y}_{g}, \mathrm{~b}_{1}, \ldots, \mathrm{b}_{n} \mid \mathrm{r}=1\right\},
$$

where

$$
\mathrm{r}=\mathrm{x}_{1} \mathrm{y}_{1} \mathrm{x}_{1}^{-1} \mathrm{y}_{1}^{-1} \cdots \mathrm{x}_{g} \mathrm{y}_{g} \mathrm{x}_{g}^{-1} \mathrm{y}_{g}^{-1} \mathrm{~b}_{1} \cdots \mathrm{b}_{n}=\Pi\left[\mathrm{x}_{i}, \mathrm{y}_{i}\right] \Pi \mathrm{b}_{j} .
$$

Then with respect to the Fox derivatives and this presentation [Ki] shows

$$
\begin{aligned}
\partial_{2} Z_{\mathrm{r}} & =\sum_{j=1}^{n} \mathrm{~b}_{j} \text { where } \\
Z_{\mathrm{r}} & =\sum_{i=1}^{g}\left(\frac{\partial \mathrm{r}}{\partial \mathrm{x}_{i}}, \mathrm{x}_{i}\right)+\left(\frac{\partial \mathrm{r}}{\partial \mathrm{y}_{i}}, \mathrm{y}_{i}\right)+\sum_{j=1}^{n}\left(\frac{\partial \mathrm{r}}{\partial \mathrm{b}_{j}}, \mathrm{~b}_{j}\right) .
\end{aligned}
$$


Consequently, he refers to $Z_{\mathrm{r}}$ as the fundamental relative cycle, since

$$
Z_{\mathrm{r}}-\sum_{j=1}^{n}\left(\frac{\partial \mathrm{r}}{\partial \mathrm{b}_{j}}, \mathrm{~b}_{j}\right)
$$

is the fundamental cycle when $n=0$, and consequently $\left[Z_{\mathrm{r}}\right]$ is a generator of $H_{2}\left(\mathrm{~F}_{r},\left\{\mathrm{~b}_{1}, \ldots, \mathrm{b}_{n}\right\} ; \mathbb{Z}\right) \cong \mathbb{Z}$ (see [GHJW]).

3.4. Parabolic cocycles. Let $\mathrm{F}_{r}^{i} \subset \mathrm{F}_{r}$ be the cyclic subgroup generated by the boundary curve $\mathrm{b}_{i}$.

Definition 9. The set of parabolic cocycles, $Z_{\mathrm{par}}^{1}\left(\mathrm{~F}_{r} ; \mathfrak{g}_{\mathrm{Ad}_{\rho}}\right) \subset Z^{1}\left(\mathrm{~F}_{r} ; \mathfrak{g}_{\mathrm{Ad}_{\rho}}\right)$, is defined by $f \in Z_{\mathrm{par}}^{1}\left(\mathrm{~F}_{r} ; \mathfrak{g}_{\mathrm{Ad}_{\rho}}\right)$ if and only if $f_{i}=\left.f\right|_{\mathrm{F}_{r}^{i}} \in B^{1}\left(\mathrm{~F}_{r}^{i}, \mathfrak{g}_{\mathrm{Ad}_{\rho}}\right)$ for all $1 \leq i \leq n$.

It is shown in Ki that

$$
B^{1}\left(\mathrm{~F}_{r} ; \mathfrak{g}_{\mathrm{Ad}_{\rho}}\right) \subset Z_{\mathrm{par}}^{1}\left(\mathrm{~F}_{r} ; \mathfrak{g}_{\mathrm{Ad}_{\rho}}\right) \subset Z^{1}\left(\mathrm{~F}_{r} ; \mathfrak{g}_{\mathrm{Ad}_{\rho}}\right) .
$$

So for $[\rho] \in \mathfrak{R}^{\text {reg }} / / \mathfrak{G}=\mathfrak{X}^{\text {reg }}$, we have

$$
H_{\mathrm{par}}^{1}\left(\mathrm{~F}_{r}, \mathfrak{g}_{\mathrm{Ad}_{\rho}}\right)=Z_{\mathrm{par}}^{1}\left(\mathrm{~F}_{r} ; \mathfrak{g}_{\mathrm{Ad}_{\rho}}\right) / B^{1}\left(\mathrm{~F}_{r} ; \mathfrak{g}_{\mathrm{Ad}_{\rho}}\right) \subset T_{[\rho]}(\mathfrak{X}) .
$$

In other words, $H_{\mathrm{par}}^{1}\left(\mathrm{~F}_{r}, \mathfrak{g}_{\mathrm{Ad}_{\rho}}\right)$ is the set of tangents that are zero on the boundary; that is, the tangents to representations with constant boundary value.

Proposition 10. $\mathcal{X}$ is foliated by the fibers of the boundary map.

Proof. The distribution $H_{\mathrm{par}}^{1}\left(\mathrm{~F}_{r}, \mathfrak{g}_{\mathrm{Ad}}\right) \subset T_{[\rho]}\left(\mathfrak{X}^{\text {reg }}\right)$ is exactly the tangents to curves in $\mathfrak{F} \cap \mathfrak{X}^{\text {reg }}$.

On the other hand, let $\rho \mapsto \operatorname{tr}(\rho(\mathrm{w}))$ for a fixed word w be a "word map." The image of sufficiently many such maps (necessarily finite) determines $[\rho]$. Then holding the boundary values fixed gives functions in $\mathbb{C}[\mathfrak{F}] \subset \mathbb{C}[\mathfrak{X}]$. As the boundary values are deformed to a different leaf, the word map is likewise deformed. Therefore, the word maps generate a family of smooth invariant vector fields that generate the distribution.

Consequently, the Stefan-Sussmann theorem (see page 17 in [DZ] implies that $\mathfrak{X}^{\text {reg }} \subset \mathcal{X}$ is foliated by $\mathfrak{F} \cap \mathfrak{X}^{\text {reg }}$. But since $\mathfrak{X}^{\text {reg }}$ is an open dense set and all vector fields corresponding to the distribution are globally defined and continuous on $\mathfrak{X}$ and non-singular on $\mathcal{X}$, they can be extended to a foliation on $\mathcal{X}$, with leaves given by $\mathcal{X} \cap \mathfrak{F}$. We note that the leaves from the submersive points of $\mathfrak{b}, \mathcal{F}$, are dense in $\mathcal{X} \cap \mathfrak{F}$.

3.5. (2,0)-form on symplectic leaves. In [GHJW], it is shown that $\omega$ from the following commutative diagram defines a symplectic form on $\mathfrak{F} \cap \mathfrak{X}^{\text {reg }}$ :

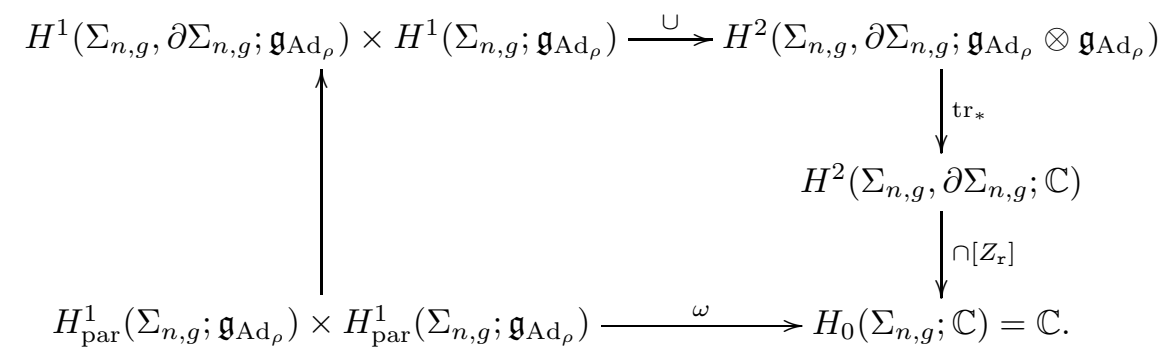


We note that in the above diagram $H_{\mathrm{par}}^{1}\left(\Sigma_{n, g} ; \mathfrak{g}_{\mathrm{Ad}}\right)$ arises from

$$
j: H^{1}\left(\Sigma_{n, g}, \partial \Sigma_{n, g} ; \mathfrak{g}_{\operatorname{Ad}_{\rho}}\right) \longrightarrow H^{1}\left(\Sigma_{n, g} ; \mathfrak{g}_{\mathrm{Ad}_{\rho}}\right),
$$

as the right factor by

$$
\operatorname{im}(j)=H_{\mathrm{par}}^{1}\left(\Sigma_{n, g} ; \mathfrak{g}_{\mathrm{Ad}_{\rho}}\right) \subset H^{1}\left(\Sigma_{n, g} ; \mathfrak{g}_{\mathrm{Ad}_{\rho}}\right)
$$

and as the left factor by

$$
H_{\text {par }}^{1}\left(\Sigma_{n, g} ; \mathfrak{g}_{\mathrm{Ad}_{\rho}}\right) \cong H^{1}\left(\Sigma_{n, g}, \partial \Sigma_{n, g} ; \mathfrak{g}_{\mathrm{Ad}_{\rho}}\right) / \operatorname{ker}(j) .
$$

Also, the map is well defined since, with respect to the cup product, $\operatorname{ker}(j)$ is orthogonal to $\operatorname{im}(j)$. Lastly, the (relative) group cohomology is isomorphic to the singular cohomology since we are working with a $K(\pi, 1)$ space.

It is clear from its definition that $\omega$ is well-defined, bilinear, and skew-symmetric, and that for all vector fields $\xi_{1}$ and $\xi_{2}$ taking values in $H_{\mathrm{par}}^{1}\left(\mathrm{~F}_{r}, \mathfrak{g}_{\mathrm{Ad}_{\rho}}\right)$,

$$
\omega\left(\xi_{1}, \xi_{2}\right): \mathfrak{X}^{r e g} \rightarrow H_{\mathrm{par}}^{1}\left(\mathrm{~F}_{r}, \mathfrak{g}_{\mathrm{Ad}_{\rho}}\right)^{\times 2} \rightarrow \mathbb{C}
$$

is an element of $\mathbb{C}[\mathfrak{X}]$ and thus smooth. Moreover, since the trace pairing is nondegenerate, $\omega$ is as well.

Consequently, it is a $(2,0)$-form. For it to be symplectic, one must check that it is closed. This is not at all obvious, and there are many differing proofs. See GHJW for a brief history and a self-contained algebraic proof.

Subsequently, using this form with Proposition 10 establishes

Corollary 11. The smooth leaves of $\mathfrak{X}$ are symplectic, and hence $\mathcal{X}$ is a Poisson manifold and $\mathfrak{X}$ is a Poisson variety (as defined at the beginning of this section).

Moreover, having the symplectic form in terms of cup and cap products allows one to verify that the Poisson bracket formula derived in G5] for closed surfaces directly generalizes to surfaces with boundary. In [Ki] this is not possible, since although he defines the 2 -form and verifies all requisite properties to establish that it is symplectic, he does not show it arises from cup and cap products. This is one of the many hallmarks of [GHJW].

Comment 12. It is worth noting that the construction of $\omega$ and the proof of its properties in [GHJW] are carried out with respect to the ground field $\mathbb{R}$. However, both its construction and the proof of it properties (in particular, closure) are done algebraically with respect to group cohomology. Consequently, the ground field may be replaced by $\mathbb{C}$ and, in particular, the proof that the form is closed remains unchanged.

Comment 13. The reader will notice that the fact $\mathfrak{G}=\mathrm{SL}(m, \mathbb{C})$ was not used to construct the 2-form; consequently much of the above discussion generalizes to character varieties defined over any reductive linear algebraic group. However, in this generality we lose many of the explicit facts concerning singularities, dimensions, generators, etc. that depend on the choice of $\mathfrak{G}$.

\section{Goldman's BRACKet formula}

In this section we prove that Goldman's bracket formula extends to surfaces with boundary. The important ideas that follow are those of Goldman (see G5]); we only apply the results of [GHJW] to his proof. 
Let $\Sigma$ be an compact, connected, oriented surface, and let $\alpha, \beta$ be immersed curves in $\Sigma$ representing elements in $\pi_{1}(\Sigma)$ (in a generic position). Denote the set of (transverse) double point intersections of $\alpha$ and $\beta$ by $\alpha \cap \beta$, and the oriented intersection number of $\alpha$ and $\beta$ at $p \in \alpha \cap \beta$ by $\epsilon(p, \alpha, \beta)$. Additionally, let $\alpha_{p} \in$ $\pi_{1}(\Sigma, p)$ represent the curve $\alpha$ based at $p$.

Then [G5] proves the following theorem for closed surfaces.

Theorem 14 (Goldman). Let $\mathbb{C}[\mathfrak{X}]$ be the coordinate ring of the $\mathrm{SL}(m, \mathbb{C})$ character variety of a closed surface group. Then

$$
\{\operatorname{tr}(\rho(\alpha)), \operatorname{tr}(\rho(\beta))\}=\sum_{p \in \alpha \cap \beta} \epsilon(p, \alpha, \beta)\left(\operatorname{tr}\left(\rho\left(\alpha_{p} \beta_{p}\right)\right)-(1 / m) \operatorname{tr}(\rho(\alpha)) \operatorname{tr}(\rho(\beta))\right)
$$

defines a Lie bracket on $\mathbb{C}[\mathfrak{X}]$ that is a derivation; in other words, a Poisson bracket.

The construction is in terms of the symplectic form for the case of closed surfaces which is likewise in terms of cohomology. Applying the $(2,0)$-form $\omega$ discussed above to the case of surfaces with boundary, we will see this same formula for the bracket remains valid on the leaves $\mathfrak{F}$.

Heuristically, this is not surprising. The reason is the bracket is summed over intersections of cycles. The boundary components are disjoint and so bracket to zero. Such functions that "Poisson commute" or bracket to zero with all other functions are called Casimirs. In parabolic cohomology these are eliminated, and so we remove the Casimirs. A bracket with no Casimirs is symplectic, so if these are the only Casimirs, the formula is valid.

4.1. Proof of the bracket formula. It is the purpose of this section to prove

Theorem 15. Let $\mathbb{C}[\mathfrak{X}]$ be the coordinate ring of the $\mathrm{SL}(m, \mathbb{C})$ character variety of a surface group having non-empty boundary. Then

$$
\{\operatorname{tr}(\rho(\alpha)), \operatorname{tr}(\rho(\beta))\}=\sum_{p \in \alpha \cap \beta} \epsilon(p, \alpha, \beta)\left(\operatorname{tr}\left(\rho\left(\alpha_{p} \beta_{p}\right)\right)-(1 / m) \operatorname{tr}(\rho(\alpha)) \operatorname{tr}(\rho(\beta))\right)
$$

defines a Lie bracket on $\mathbb{C}[\mathfrak{X}]$ that is a derivation; in other words, a Poisson bracket.

The Poisson bracket of two functions on a symplectic leaf of a Poisson manifold is given by $\left\{f_{\alpha}, f_{\beta}\right\}=\omega\left(H f_{\alpha}, H f_{\beta}\right)$, where $H f_{\alpha}$ is the Hamiltonian vector field associated to $f_{\alpha}$. We will first describe the Hamiltonians in terms of cycles and then use the fact that the 2 -form is in terms of cup and cap products to relate these to intersection pairings. This will prove the formula to be valid.

We begin by setting out the terms we will need for the discussion and then stating a lemma that will reduce the proof of Theorem 15] to a computation.

Let $f: \mathfrak{G} \rightarrow \mathbb{C}$ be an invariant function in $\mathbb{C}[\mathfrak{G}]$. Then $d f$ is a 1 -form on $\mathfrak{G}$. Let $\mathbf{A} \in \mathfrak{G}$; then $d f(\mathbf{A}) \in T_{\mathbf{A}}^{*}(\mathfrak{G})$ and extends to a right invariant 1-form $F^{*}(\mathbf{A}) \in \mathfrak{g}^{*}$. Here $F^{*}: \mathfrak{G} \rightarrow \mathfrak{g}^{*}$ is defined (for $X \in \mathfrak{g}$ ) by

$$
F^{*}(\mathbf{A}):\left.X \mapsto \frac{d}{d t}\right|_{t=0} f(\exp (t X) \mathbf{A}) .
$$

The trace is a non-degenerate symmetric bilinear form $\mathfrak{g} \times \mathfrak{g} \rightarrow \mathbb{C}$. Thus as $\mathfrak{G}$-modules under the adjoint representation, $\mathfrak{g} \cong \mathfrak{g}^{*}$ by $\operatorname{tr}_{*}: X \mapsto \operatorname{tr}\left(X_{-}\right)$. Let 
$F=\operatorname{tr}_{*}^{-1} F^{*}: \mathfrak{G} \rightarrow \mathfrak{g}^{*} \rightarrow \mathfrak{g}$. Then for $f(\mathbf{A})=\operatorname{tr}(\mathbf{A})$ we have

$$
\begin{gathered}
\operatorname{tr}(X F(\mathbf{A}))=\operatorname{tr}_{*} F(\mathbf{A})(X)=F^{*}(\mathbf{A})(X)=\left.\frac{d}{d t}\right|_{t=0} f(\exp (t X) \mathbf{A}) \\
=\operatorname{tr}(X \mathbf{A})=\operatorname{tr}(X \mathbf{A})-\frac{1}{m} \operatorname{tr}(\mathbf{A}) \operatorname{tr}(X)=\operatorname{tr}\left(X\left(\mathbf{A}-\frac{1}{m} \operatorname{tr}(\mathbf{A}) \mathbb{I}\right)\right),
\end{gathered}
$$

and so because the trace is non-degenerate,

$$
F(\mathbf{A})=\mathbf{A}-\frac{1}{m} \operatorname{tr}(\mathbf{A}) \mathbb{I} .
$$

Now consider $\alpha \in \pi_{1}(\Sigma, *)$, where we choose a representative that is an immersed curve in $\Sigma$ that intersects itself only at transverse double points. Let $\rho: \pi_{1}(\Sigma, p) \rightarrow$ $\mathfrak{G}$ represent $[\rho] \in \mathfrak{X}$ and $\alpha_{p} \in \pi_{1}(\Sigma, p)$ correspond to $\alpha$ where $p$ is a simple point (not a point of self-intersection).

The Hamiltonian vector field of the Goldman function $f_{\alpha}(\rho)=\operatorname{tr}(\rho(\alpha))$, a map $\mathfrak{X} \rightarrow \mathbb{C}$, is given implicitly by $d f_{\alpha}=\omega\left(H f_{\alpha}, \ldots\right)$. In other words, $H f_{\alpha}=\omega_{*}^{-1}\left(d f_{\alpha}\right)$, where $\omega_{*}$ is the isomorphism

$$
H_{\mathrm{par}}^{1}\left(\mathrm{~F}_{r}, \mathfrak{g}_{\mathrm{Ad}_{\rho}}\right) \rightarrow H_{\mathrm{par}}^{1}\left(\mathrm{~F}_{r}, \mathfrak{g}_{\mathrm{Ad}_{\rho}}\right)^{*}
$$

given by $v \mapsto \omega(, v)$. The chain $\alpha_{p} \otimes F\left(\rho\left(\alpha_{p}\right)\right)$ is a cycle since $\operatorname{Ad}_{\rho}\left(\alpha_{p}\right) F\left(\rho\left(\alpha_{p}\right)\right)=$ $F\left(\rho\left(\alpha_{p}\right)\right)$, and consequently represents a relative cycle in $H_{1}\left(\mathrm{~F}_{r},\left\{\mathrm{~b}_{1}, \ldots, \mathrm{b}_{n}\right\} ; \mathfrak{g}_{\mathrm{Ad}_{\rho}}\right)$ since non-trivial cycles are relative cycles.

The following lemma describes how the Hamiltonian relates to this relative cycle.

Lemma 16. The relative cycle $\alpha_{p} \otimes F\left(\rho\left(\alpha_{p}\right)\right)$ is equal to the cap product of the Hamiltonian $H f_{\alpha}$ with the fundamental relative cycle $Z_{\mathrm{r}}$. In other words, $\alpha_{p} \otimes$ $F\left(\rho\left(\alpha_{p}\right)\right)=H f_{\alpha} \cap\left[Z_{r}\right]$.

Assuming Lemma 16] we now prove Theorem 15.

Proof of Theorem 15. To derive the bracket formula, we will need to use the intersection pairing of cycles. Since we are working with $K(\pi, 1)$ we may pass from group cohomology to singular cohomology, work with intersection theory, and then return to group cohomology.

We quickly remind the reader of facts concerning intersection pairings which may be found on page 367 in $[\mathrm{Br}$. Let $D$ be the inverse of the Poincare duality map

$$
D: H_{1}\left(\Sigma ; \mathfrak{g}_{\mathrm{Ad}_{\rho}}\right) \rightarrow H^{1}\left(\Sigma, \partial \Sigma ; \mathfrak{g}_{\mathrm{Ad}_{\rho}}\right) ;
$$

that is, $D(u) \cap\left[Z_{r}\right]=u$. The intersection pairing

$$
\bullet: H_{1}\left(\Sigma, \partial \Sigma ; \mathfrak{g}_{\mathrm{Ad}_{\rho}}\right) \times H_{1}\left(\Sigma, \partial \Sigma ; \mathfrak{g}_{\mathrm{Ad}_{\rho}}\right) \rightarrow H_{2}\left(\Sigma, \partial \Sigma ; \mathfrak{g}_{\mathrm{Ad}_{\rho}}\right)
$$

is defined by

$$
u \bullet v=(D(v) \cup D(u)) \cap\left[Z_{r}\right] .
$$

It has the geometric consequence of being equal to the sum taken over transverse intersections of 1-cycles with coefficients. Precisely,

$$
(\alpha \otimes F) \bullet(\beta \otimes F)=\sum_{p \in \alpha \cap \beta} \epsilon(p, \alpha, \beta) F\left(\alpha_{p}\right) \otimes F\left(\beta_{p}\right) .
$$



16

We now compute the Poisson bracket of two invariant functions using Lemma

$$
\begin{aligned}
\left\{f_{\alpha}, f_{\beta}\right\}= & \omega\left(H f_{\alpha}, H f_{\beta}\right) \\
= & \operatorname{tr}_{*}\left(H f_{\alpha} \cup H f_{\beta}\right) \cap\left[Z_{r}\right] \\
= & \operatorname{tr}_{*}\left(\left(D\left(H f_{\alpha} \cap\left[Z_{r}\right]\right)\right) \cup\left(D\left(H f_{\beta} \cap\left[Z_{r}\right]\right)\right) \cap\left[Z_{r}\right]\right) \\
= & \operatorname{tr}_{*}\left(\left(H f_{\alpha} \cap\left[Z_{r}\right]\right) \bullet\left(H f_{\beta} \cap\left[Z_{r}\right]\right)\right) \\
= & \operatorname{tr}_{*}\left(\alpha \otimes F\left(\rho\left(\alpha_{p}\right)\right) \bullet \beta \otimes F\left(\rho\left(\beta_{p}\right)\right)\right) \\
= & \sum_{p \in \alpha \cap \beta} \epsilon(p, \alpha, \beta) \operatorname{tr}\left(F\left(\rho\left(\alpha_{p}\right)\right) F\left(\rho\left(\beta_{p}\right)\right)\right) \\
= & \sum_{p \in \alpha \cap \beta} \epsilon(p, \alpha, \beta) \operatorname{tr}\left(\left(\rho\left(\alpha_{p}\right)-(1 / m) \operatorname{tr}\left(\rho\left(\alpha_{p}\right) \mathbb{I}\right)\left(\rho\left(\beta_{p}\right)\right.\right.\right. \\
& \left.\left.-(1 / m) \operatorname{tr}\left(\rho\left(\beta_{p}\right)\right) \mathbb{I}\right)\right) \\
= & \sum_{p \in \alpha \cap \beta} \epsilon(p, \alpha, \beta)\left(\operatorname{tr}\left(\rho\left(\alpha_{p} \beta_{p}\right)\right)-(1 / m) \operatorname{tr}\left(\rho\left(\alpha_{p}\right)\right) \operatorname{tr}\left(\rho\left(\beta_{p}\right)\right)\right) .
\end{aligned}
$$

This concludes the proof of the bracket formula.

It now remains to prove Lemma 16

Proof of Lemma 16. Let $[u] \in H_{\mathrm{par}}^{1}\left(\mathrm{~F}_{r}, \mathfrak{g}_{\mathrm{Ad}_{\rho}}\right)$, so $u \in Z_{\mathrm{par}}^{1}\left(\mathrm{~F}_{r}, \mathfrak{g}_{\mathrm{Ad}_{\rho}}\right)$, and let $\rho_{t}$ be a path in $\mathfrak{R}$ so $u$ is a tangent. Then

$$
\left(d f_{\alpha}\right)_{\rho}:\left.[u] \mapsto \frac{d}{d t}\right|_{t=0} f_{\alpha}\left(\rho_{t}\left(\alpha_{p}\right)\right)=F^{*}\left(\rho\left(\alpha_{p}\right)\right)\left(u\left(\alpha_{p}\right)\right)=\operatorname{tr}\left(F\left(\rho\left(\alpha_{p}\right)\right) u\left(\alpha_{p}\right)\right) .
$$

We will now define a number of mappings which we will show are related by a commutative diagram; the lemma will follow.

The trace gives a duality pairing dp $: \mathfrak{g} \rightarrow \mathfrak{g}^{*}$ given by $u \mapsto \operatorname{tr}\left(u_{-}\right)$which pushes forward to

$$
\mathrm{dp}_{*}: H^{1}\left(\mathrm{~F}_{r} ; \mathfrak{g}_{\mathrm{Ad}_{\rho}}\right) \rightarrow H^{1}\left(\mathrm{~F}_{r} ; \mathfrak{g}_{\mathrm{Ad}_{\rho}}^{*}\right) .
$$

Consequently, $\left(\operatorname{dp}_{*}\right)^{*}: H_{\text {par }}^{1}\left(\mathrm{~F}_{r} ; \mathfrak{g}_{\text {Ad }}^{*}\right)^{*} \rightarrow H_{\text {par }}^{1}\left(\mathrm{~F}_{r} ; \mathfrak{g}_{\mathrm{Ad}_{\rho}}\right)^{*}$ defined by $v \mapsto v\left(\operatorname{dp}_{*}\left(\_\right)\right)$ is an isomorphism.

Using the Kroneker product define

$$
\text { kp : } H_{1}\left(\mathrm{~F}_{r},\left\{\mathrm{~b}_{1}, \ldots, \mathrm{b}_{n}\right\} ; \mathfrak{g}_{\mathrm{Ad}_{\rho}}\right) \rightarrow H_{\mathrm{par}}^{1}\left(\mathrm{~F}_{r} ; \mathfrak{g}_{\mathrm{Ad}}^{*}\right)^{*}
$$

by $\alpha \otimes F\left(\rho\left(\alpha_{p}\right)\right) \mapsto \operatorname{tr}_{*}\left\langle\operatorname{dp}_{*}^{-1}\left(\_\right), \alpha \otimes F\left(\rho\left(\alpha_{p}\right)\right)\right\rangle$. We claim lp is well-defined. Let $u$ be a parabolic cycle, $\beta$ a 2-chain, and $\gamma$ a 1-chain on the boundary. Then

$u(\alpha \otimes F+\partial \beta+\gamma)=u(\alpha \otimes F)+u(\partial \beta)+u(\gamma)=u(\alpha \otimes F)+\delta u(\beta)+0=u(\alpha \otimes F)$ because $\delta u=0$ since $u$ is a cycle, and $u(\gamma)=0$ since $u$ is parabolic. This verifies that $\mathrm{kp}$ is well-defined.

Lastly, in terms of Poincaré duality let PD : $H_{\mathrm{par}}^{1}\left(\mathrm{~F}_{r} ; \mathfrak{g}_{\mathrm{Ad}_{\rho}}\right) \rightarrow H_{\mathrm{par}}^{1}\left(\mathrm{~F}_{r} ; \mathfrak{g}_{\mathrm{Ad}_{\rho}}^{*}\right)^{*}$ be the isomorphism

$$
u \mapsto \operatorname{tr}_{*}\left(\mathrm{dp}_{*}^{-1}\left(\_\right) \cup u\right) \cap\left[Z_{r}\right] .
$$


In these terms, we have

Lemma 17. The following diagram commutes:

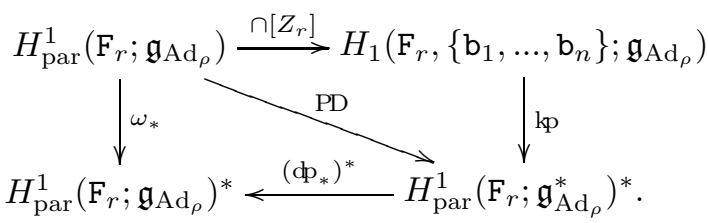

We defer proving Lemma 17 until after the proof of Lemma 16

To complete the proof of Lemma 16 we begin with a relative cycle $\alpha \otimes F$ starting in the upper right corner of the diagram:

$$
\begin{aligned}
\left(\mathrm{dp}_{*}\right)^{*}(\operatorname{kp}(\alpha \otimes F)) & =\left(\mathrm{dp}_{*}\right)^{*} \operatorname{tr}_{*}\left\langle\mathrm{dp}_{*}^{-1}\left(\_\right), \alpha \otimes F\right\rangle \\
& =\operatorname{tr}_{*}\left\langle\operatorname{dp}_{*}^{-1} \mathrm{dp}_{*}\left(\_\right), \alpha \otimes F\right\rangle=\operatorname{tr}_{*}\left\langle \_, \alpha \otimes F\right\rangle=d f_{\alpha} .
\end{aligned}
$$

However, since $\left(\mathrm{dp}_{*}\right)^{*}$ is an isomorphism and $\mathrm{kp}$ is injective on the image of $H_{\text {par }}^{1}\left(\mathrm{~F}_{r}, \mathfrak{g}_{\mathrm{Ad}_{\rho}}\right)$ under $\cap\left[Z_{\mathrm{r}}\right]$, we have

$$
\alpha_{p} \otimes F\left(\rho\left(\alpha_{p}\right)\right)=\operatorname{kp}^{-1}\left(\left(\mathrm{dp}_{*}\right)^{*}\right)^{-1}\left(d f_{\alpha}\right)=\left(\omega_{*}\right)^{-1}\left(d f_{\alpha}\right) \cap\left[Z_{r}\right]=H f_{\alpha} \cap\left[Z_{r}\right],
$$

by commutativity of the above diagram. Note that $\mathrm{kp}$ is injective on the image of $H_{\text {par }}^{1}\left(\mathrm{~F}_{r}, \mathfrak{g}_{\mathrm{Ad}_{\rho}}\right)$ under $\cap\left[Z_{\mathrm{r}}\right]$ because PD is an isomorphism and by commutativity. This completes the proof.

It remains to prove Lemma 17

Proof of Lemma 17. First we show the lower triangle commutes:

$$
\begin{aligned}
\left(\mathrm{dp}_{*}\right)^{*} \operatorname{PD}(v) & =\left(\mathrm{dp}_{*}\right)^{*}\left(\operatorname{tr}_{*}\left(\mathrm{dp}_{*}^{-1}\left(\_\right) \cup v\right) \cap\left[Z_{r}\right]\right)=\operatorname{tr}_{*}\left(\mathrm{dp}_{*}^{-1} \mathrm{dp}_{*}\left(\_\right) \cup v\right) \cap\left[Z_{r}\right] \\
& =\omega\left(\_, v\right)=\omega_{*}(v) .
\end{aligned}
$$

Second, we show the upper triangle commutes (see [B] page 113):

$$
\begin{aligned}
\operatorname{kp}\left(v \cap\left[Z_{r}\right]\right) & =\operatorname{tr}_{*}\left\langle\mathrm{dp}_{*}^{-1}\left(\_\right), v \cap\left[Z_{r}\right]\right\rangle=\left\langle\operatorname{tr}_{*}\left(\operatorname{dp}_{*}^{-1}\left(\_\right) \cup v\right),\left[Z_{r}\right]\right\rangle \\
& =\operatorname{tr}_{*}\left(\mathrm{dp}_{*}^{-1}\left(\_\right) \cup v\right) \cap\left[Z_{r}\right]=\operatorname{PD}(v) .
\end{aligned}
$$

Comment 18. The above argument can be generalized to any reductive linear algebraic group. Such groups have a symmetric, non-degenerate bilinear form $\mathfrak{B}$ on their Lie algebra that is invariant under the adjoint representation. In the above argument this was the trace form. To generalize the argument replace $\operatorname{tr}_{*}$ by $\mathfrak{B}_{*}$ and replace the explicit form of $F$ by its general form defined by $\mathfrak{B}(F(\mathbf{A}), X)=$ $\left.\frac{d}{d t}\right|_{t=0} f(\exp (t X) \mathbf{A})$. In special cases, like $\mathfrak{G}=\mathrm{SL}(m, \mathbb{C}), F$ can be computed explicitly as we did above. See [G5] for further details.

Comment 19. Since the bracket formula depends on a choice of orientation of the surface, we immediately have that switching the orientation of a surface multiplies the Poisson structure (the bi-vector) by -1 .

We conclude this section by stating explicitly how the Poisson structure distinguishes the moduli of surface group representations where the algebraic structure alone cannot. 
Theorem 20. Let $\Sigma$ and $\Sigma^{\prime}$ be compact, connected, orientable surfaces with boundary, and let $\mathfrak{X}(\Sigma)$ and $\mathfrak{X}\left(\Sigma^{\prime}\right)$ be the moduli of surface group representations into $\mathrm{SL}(m, \mathbb{C})$. Then:

(1) $\mathfrak{X}(\Sigma)$ and $\mathfrak{X}\left(\Sigma^{\prime}\right)$ are isomorphic as varieties if and only if $\chi(\Sigma)=\chi\left(\Sigma^{\prime}\right)$.

(2) $\mathfrak{X}(\Sigma)$ and $\mathfrak{X}\left(\Sigma^{\prime}\right)$ are isomorphic as Poisson varieties if and only if $\Sigma$ and $\Sigma^{\prime}$ are homeomorphic.

Proof. $\chi(\Sigma)=\chi\left(\Sigma^{\prime}\right)$ if and only if the ranks of their fundamental groups are equal, which implies $\mathfrak{X}(\Sigma)$ and $\mathfrak{X}\left(\Sigma^{\prime}\right)$ are isomorphic as varieties. On the other hand, if they are isomorphic as varieties, then they must share the same transcendence degree. The transcendence degree is computed to be $-\chi(\Sigma)\left(m^{2}-1\right)$ in general. Consequently, they have the same Euler characteristic.

Observe $\Sigma$ and $\Sigma^{\prime}$ are homeomorphic if and only if they have the same number of boundaries and the same genus. Their moduli are equivalent as Poisson varieties if and only if they are isomorphic as varieties and there is a Lie algebra isomorphism of their coordinate rings. With the same boundaries and genus, the moduli are certainly isomorphic by our previous work. Moreover, there is certainly not a Lie algebra isomorphism if the Casimir subalgebras are different. Since the bracket sums over intersections of cycles and in a surface the only cycles which necessarily are parallel to all other homotopy classes of cycles are the boundaries, the number of boundaries $n$ gives $n(m-1)$ generically independent generators of the Casimir subalgebra (since the boundary mapping is generically submersive). Consequently, if $\mathfrak{X}(\Sigma)$ and $\mathfrak{X}\left(\Sigma^{\prime}\right)$ are isomorphic as Poisson varieties and $\Sigma$ and $\Sigma^{\prime}$ are not homeomorphic, we get a contradiction. Equivalently, we have that the symplectic leaves have generic dimensions in terms of the number of boundaries. So again, if the surfaces are not homeomorphic we have a contradiction, since the Poisson structures are equivalent if the corresponding symplectic foliations are equivalent. On the other hand, if $\Sigma$ and $\Sigma^{\prime}$ are homeomorphic the intersection pairing on cohomology will be equivalent. Since the Poisson bracket is in terms of intersections the moduli will have equivalent brackets. This will allow for a Lie algebra isomorphism of their coordinate rings.

\section{Structure of $\mathbb{C}\left[\mathfrak{G}^{\times 2} / / \mathfrak{G}\right]$}

We now restrict our attention to $m=3$; that is, to $\mathrm{SL}(3, \mathbb{C})$ character varieties. The reason for this is that an explicit description of the Poisson bracket requires knowledge of the variety. However, an explicit description of the algebraic structure is not known in general. In fact, the only general formulation is for $\operatorname{SL}(2, \mathbb{C})$. In an upcoming paper we explore more generally the Poisson structure for $\mathrm{SL}(2, \mathbb{C})$ character varieties. For $\operatorname{SL}(3, \mathbb{C})$ character varieties the ideal of relations defining the character variety is only known for rank 1 and 2 free groups. In this section we review results in $[\mathrm{L}$ that we will need to characterize the Poisson structures on $\mathrm{SL}(3, \mathbb{C})$ character varieties of Euler characteristic -1 surfaces.

5.1. Minimal generators. From Section 2 we know that $\mathbb{C}[\mathfrak{X}]$ is generated by traces of words in generic matrices of word length no greater than 6 . The following lemma may be found in $[\mathrm{L}$. 
Lemma 21. $\mathbb{C}[\mathfrak{G} \times \mathfrak{G}]^{\mathfrak{G}}$ is minimally generated by

$$
\begin{aligned}
& \operatorname{tr}\left(\mathbf{X}_{1}\right), \operatorname{tr}\left(\mathbf{X}_{2}\right), \operatorname{tr}\left(\mathbf{X}_{1} \mathbf{X}_{2}\right), \operatorname{tr}\left(\mathbf{X}_{1} \mathbf{X}_{2}^{-1}\right), \operatorname{tr}\left(\mathbf{X}_{1}^{-1}\right), \\
& \operatorname{tr}\left(\mathbf{X}_{2}^{-1}\right), \operatorname{tr}\left(\mathbf{X}_{1}^{-1} \mathbf{X}_{2}^{-1}\right), \quad \operatorname{tr}\left(\mathbf{X}_{1}^{-1} \mathbf{X}_{2}\right), \operatorname{tr}\left(\mathbf{X}_{1} \mathbf{X}_{2} \mathbf{X}_{1}^{-1} \mathbf{X}_{2}^{-1}\right) .
\end{aligned}
$$

Since we are working with invariants of pairs of $3 \times 3$ unimodular matrices, the Krull dimension is 8 . With 9 generators, we expect one relation.

5.2. Hyper-surface in $\mathbb{C}^{9}$. With a minimal generating set, it remains to describe the relations in terms of these generators.

Let

$$
\bar{R}=\mathbb{C}\left[t_{(1)}, t_{(-1)}, t_{(2)}, t_{(-2)}, t_{(3)}, t_{(-3)}, t_{(4)}, t_{(-4)}, t_{(5)}, t_{(-5)}\right]
$$

be the complex polynomial ring freely generated by $\left\{t_{( \pm i)}, 1 \leq i \leq 5\right\}$, and let

$$
R=\mathbb{C}\left[t_{(1)}, t_{(-1)}, t_{(2)}, t_{(-2)}, t_{(3)}, t_{(-3)}, t_{(4)}, t_{(-4)}\right]
$$

be its subring generated by $\left\{t_{( \pm i)}, 1 \leq i \leq 4\right\}$, so $\bar{R}=R\left[t_{(5)}, t_{(-5)}\right]$. Define the ring homomorphism

$$
R\left[t_{(5)}, t_{(-5)}\right] \stackrel{\Pi}{\longrightarrow} \mathbb{C}[\mathfrak{G} \times \mathfrak{G}]^{\mathfrak{G}}
$$

by

$$
\begin{array}{lll}
t_{(1)} \mapsto \operatorname{tr}\left(\mathbf{X}_{1}\right), & t_{(-1)} \mapsto \operatorname{tr}\left(\mathbf{X}_{1}^{-1}\right) \\
t_{(2)} \mapsto \operatorname{tr}\left(\mathbf{X}_{2}\right), & t_{(-2)} \mapsto \operatorname{tr}\left(\mathbf{X}_{2}^{-1}\right) \\
t_{(3)} \mapsto \operatorname{tr}\left(\mathbf{X}_{1} \mathbf{X}_{2}\right), & t_{(-3)} \mapsto \operatorname{tr}\left(\mathbf{X}_{1}^{-1} \mathbf{X}_{2}^{-1}\right) \\
t_{(4)} \mapsto \operatorname{tr}\left(\mathbf{X}_{1} \mathbf{X}_{2}^{-1}\right), & t_{(-4)} \mapsto \operatorname{tr}\left(\mathbf{X}_{1}^{-1} \mathbf{X}_{2}\right), \\
t_{(5)} \mapsto \operatorname{tr}\left(\mathbf{X}_{1} \mathbf{X}_{2} \mathbf{X}_{1}^{-1} \mathbf{X}_{2}^{-1}\right), & t_{(-5)} \mapsto \operatorname{tr}\left(\mathbf{X}_{2} \mathbf{X}_{1} \mathbf{X}_{2}^{-1} \mathbf{X}_{1}^{-1}\right)
\end{array}
$$

It follows from Lemma 21 that

$$
\mathbb{C}[\mathfrak{X}] \cong R\left[t_{(5)}, t_{(-5)}\right] / \operatorname{ker}(\Pi) .
$$

In other words, $\Pi$ is a surjective algebra morphism. We define

$$
\begin{gathered}
P=t_{(1)} t_{(-1)} t_{(2)} t_{(-2)}-t_{(1)} t_{(2)} t_{(-3)}-t_{(-1)} t_{(-2)} t_{(3)}-t_{(1)} t_{(-2)} t_{(-4)}-t_{(-1)} t_{(2)} t_{(4)} \\
+t_{(1)} t_{(-1)}+t_{(2)} t_{(-2)}+t_{(3)} t_{(-3)}+t_{(4)} t_{(-4)}-3
\end{gathered}
$$

and so $P \in R$. It is shown in $[\mathrm{L}$ that

$$
P-\left(t_{(5)}+t_{(-5)}\right) \in \operatorname{ker}(\Pi)
$$

Hence it follows that the composite map

$$
R\left[t_{(5)}\right] \hookrightarrow R\left[t_{(5)}, t_{(-5)}\right] \rightarrow R\left[t_{(5)}, t_{(-5)}\right] / \operatorname{ker}(\Pi)
$$

is an epimorphism. Let $I$ be the kernel of this composite map. In $[\mathrm{L}$ it is then shown that there exists $Q \in R$ so $Q-t_{(5)} t_{(-5)} \in \operatorname{ker}(\Pi)$ as well.

As a consequence we have the following lemma describing the ideal of relations defining the character variety.

Lemma 22. I is principally generated by the polynomial

$$
t_{(5)}^{2}-P t_{(5)}+Q
$$


The form of $Q$ is shown to be

$$
\begin{aligned}
& Q=9-6 t_{(1)} t_{(-1)}-6 t_{(2)} t_{(-2)}-6 t_{(3)} t_{(-3)}-6 t_{(4)} t_{(-4)}+t_{(1)}^{3}+t_{(2)}^{3}+t_{(3)}^{3}+t_{(4)}^{3} \\
& +t_{(-1)}^{3}+t_{(-2)}^{3}+t_{(-3)}^{3}+t_{(-4)}^{3}-3 t_{(-4)} t_{(-3)} t_{(-1)}-3 t_{(4)} t_{(3)} t_{(1)} \\
& -3 t_{(-4)} t_{(2)} t_{(3)}-3 t_{(4)} t_{(-2)} t_{(-3)}+3 t_{(-4)} t_{(-2)} t_{(1)}+3 t_{(4)} t_{(2)} t_{(-1)} \\
& +3 t_{(1)} t_{(2)} t_{(-3)}+3 t_{(-1)} t_{(-2)} t_{(3)}+t_{(-2)} t_{(-1)} t_{(2)} t_{(1)}+t_{(-3)} t_{(-2)} t_{(3)} t_{(2)} \\
& +t_{(-4)} t_{(-1)} t_{(4)} t_{(1)}+t_{(-4)} t_{(-2)} t_{(4)} t_{(2)}+t_{(-3)} t_{(-1)} t_{(3)} t_{(1)} \\
& +t_{(-3)} t_{(-4)} t_{(3)} t_{(4)}+t_{(-4)}^{2} t_{(-3)} t_{(-2)}+t_{(4)}^{2} t_{(3)} t_{(2)}+t_{(-1)}^{2} t_{(-2)} t_{(-4)}+t_{(1)}^{2} t_{(2)} t_{(4)} \\
& +t_{(1)} t_{(-2)}^{2} t_{(-3)}+t_{(-1)} t_{(2)}^{2} t_{(3)}+t_{(-4)} t_{(-3)} t_{(1)}^{2}+t_{(4)} t_{(3)} t_{(-1)}^{2} \\
& +t_{(-4)} t_{(2)} t_{(-3)}^{2}+t_{(4)} t_{(-2)} t_{(3)}^{2}+t_{(-1)}^{2} t_{(-3)} t_{(2)}+t_{(1)}^{2} t_{(3)} t_{(-2)} \\
& +t_{(-4)} t_{(1)} t_{(2)}^{2}+t_{(4)} t_{(-1)} t_{(-2)}^{2}+t_{(-4)} t_{(3)} t_{(-2)}^{2}+t_{(4)} t_{(-3)} t_{(2)}^{2} \\
& +t_{(1)} t_{(3)} t_{(-4)}^{2}+t_{(-1)} t_{(-3)} t_{(4)}^{2}+t_{(-1)} t_{(-4)} t_{(3)}^{2}+t_{(1)} t_{(4)} t_{(-3)}^{2}-2 t_{(-3)}^{2} t_{(-2)} t_{(-1)} \\
& -2 t_{(3)}^{2} t_{(2)} t_{(1)}-2 t_{(-4)}^{2} t_{(-1)} t_{(2)}-2 t_{(4)}^{2} t_{(1)} t_{(-2)}+t_{(-1)}^{2} t_{(-2)}^{2} t_{(-3)}+t_{(1)}^{2} t_{(2)}^{2} t_{(3)} \\
& +t_{(-4)} t_{(-1)}^{2} t_{(2)}^{2}+t_{(4)} t_{(1)}^{2} t_{(-2)}^{2}-t_{(-4)} t_{(-2)}^{2} t_{(2)} t_{(1)}-t_{(4)} t_{(2)}^{2} t_{(-2)} t_{(-1)} \\
& -t_{(-3)} t_{(1)}^{2} t_{(-1)} t_{(2)}-t_{(3)} t_{(-1)}^{2} t_{(1)} t_{(-2)}-t_{(-3)} t_{(2)}^{2} t_{(-2)} t_{(1)}-t_{(3)} t_{(-2)}^{2} t_{(2)} t_{(-1)} \\
& -t_{(-4)} t_{(-2)} t_{(-1)} t_{(1)}^{2}-t_{(4)} t_{(2)} t_{(1)} t_{(-1)}^{2}-t_{(-1)} t_{(-2)}^{3} t_{(1)}-t_{(-1)} t_{(2)}^{3} t_{(1)} \\
& -t_{(-1)}^{3} t_{(-2)} t_{(2)}-t_{(1)}^{3} t_{(-2)} t_{(2)}-t_{(-4)} t_{(-3)} t_{(-2)} t_{(-1)} t_{(2)}-t_{(4)} t_{(3)} t_{(2)} t_{(1)} t_{(-2)} \\
& -t_{(-1)} t_{(1)} t_{(2)} t_{(-4)} t_{(3)}-t_{(-1)} t_{(1)} t_{(-2)} t_{(4)} t_{(-3)}+t_{(-2)} t_{(-1)}^{2} t_{(1)}^{2} t_{(2)} \\
& +t_{(-1)} t_{(-2)}^{2} t_{(2)}^{2} t_{(1)}
\end{aligned}
$$

Lemmas 21 and 22 together imply

Theorem 23. $\mathfrak{G}^{\times 2} / / \mathfrak{G}$ is isomorphic to an affine degree 6 hyper-surface in $\mathbb{C}^{9}$, which maps onto $\mathbb{C}^{8}$ generically 2-to-1.

With the generators and relations in hand, we now briefly describe symmetry within the character variety coming from the outer automorphisms of the surface group.

5.3. Outer automorphisms. Given any $\alpha \in \operatorname{Aut}\left(\mathrm{F}_{2}\right)$, we define $a_{\alpha} \in \operatorname{End}(\mathbb{C}[\mathfrak{X}])$ by extending the following mapping:

$$
a_{\alpha}(\operatorname{tr}(\mathbf{W}))=\operatorname{tr}(\alpha(\mathbf{W})) .
$$

If $\alpha \in \operatorname{Inn}\left(\mathrm{F}_{2}\right)$, then there exists $\mathrm{u} \in \mathrm{F}_{2}$ so for all $\mathrm{w} \in \mathrm{F}_{2}$,

$$
\alpha(\mathrm{w})=\mathrm{uwu}^{-1},
$$

which implies

$$
a_{\alpha}(\operatorname{tr}(\mathbf{W}))=\operatorname{tr}\left(\mathbf{U} \mathbf{W} \mathbf{U}^{-1}\right)=\operatorname{tr}(\mathbf{W})
$$


Thus $\operatorname{Out}\left(\mathrm{F}_{2}\right)$ acts on $\mathbb{C}[\mathfrak{X}]$. By results in $\underline{\mathrm{MKS}}$, Out $\left(\mathrm{F}_{2}\right)$ is generated by the following mappings:

$$
\begin{gathered}
\mathfrak{t}=\left\{\begin{array}{l}
\mathrm{x}_{1} \mapsto \mathrm{x}_{2}, \\
\mathrm{x}_{2} \mapsto \mathrm{x}_{1},
\end{array}\right. \\
\mathfrak{i}_{1}=\left\{\begin{array}{l}
\mathrm{x}_{1} \mapsto \mathrm{x}_{1}^{-1}, \\
\mathrm{x}_{2} \mapsto \mathrm{x}_{2},
\end{array}\right. \\
\mathfrak{n}=\left\{\begin{array}{l}
\mathrm{x}_{1} \mapsto \mathrm{x}_{1} \mathrm{x}_{2}, \\
\mathrm{x}_{2} \mapsto \mathrm{x}_{2},
\end{array}\right.
\end{gathered}
$$

More generally for any $\alpha \in \operatorname{Out}\left(\mathrm{F}_{r}\right), \operatorname{tr}(\alpha(\mathbf{W}))$ is a polynomial in traces of words; consequently $\operatorname{Out}\left(\mathrm{F}_{r}\right)$ acts by polynomials.

Let $\mathfrak{D}$ be the subgroup generated by $\mathfrak{t}$ and $\mathfrak{i}_{1}$, and let $\mathbb{C} \mathfrak{D}$ be the corresponding group ring. Then $\mathbb{C}[\mathfrak{X}]$ is a $\mathbb{C} \mathfrak{D}$-module. It is not hard to work out that $\mathfrak{D}$ is isomorphic to the order 8 dihedral group $D_{4}$. Moreover, the projection from Theorem 23 is $\mathfrak{D}$-equivariant.

Using this action, one can establish the following succinct expressions for the polynomial relations $P$ and $Q$ (see $[\mathrm{L}]$ ).

Corollary 24. In $\mathbb{C D}$ define $\mathbb{S}_{\mathfrak{D}}=\sum_{\sigma \in \mathfrak{D}} \sigma$. Then $P=\mathbb{S}_{\mathfrak{D}}(p)-3$ and $Q=\mathbb{S}_{\mathfrak{D}}(q)+9$ where $p$ and $q$ are given by

$$
\begin{aligned}
p= & \frac{1}{8}\left(t_{(1)} t_{(-1)} t_{(2)} t_{(-2)}-4 t_{(1)} t_{(-2)} t_{(-4)}+2 t_{(1)} t_{(-1)}+2 t_{(3)} t_{(-3)}\right) \\
q= & \frac{1}{8}\left(2 t_{(-2)} t_{(-1)}^{2} t_{(1)}^{2} t_{(2)}+4 t_{(1)}^{2} t_{(2)}^{2} t_{(3)}-4 t_{(1)}^{3} t_{(-2)} t_{(2)}-8 t_{(-4)} t_{(-2)} t_{(-1)} t_{(1)}^{2}\right. \\
- & 4 t_{(4)} t_{(3)} t_{(2)} t_{(1)} t_{(-2)}+8 t_{(1)} t_{(3)} t_{(-4)}^{2}+8 t_{(-4)} t_{(1)} t_{(2)}^{2}-8 t_{(3)}^{2} t_{(2)} t_{(1)} \\
+ & 4 t_{(4)} t_{(-3)} t_{(2)}^{2}+t_{(-2)} t_{(-1)} t_{(2)} t_{(1)}+t_{(-3)} t_{(-4)} t_{(3)} t_{(4)}+4 t_{(-3)} t_{(-1)} t_{(3)} t_{(1)} \\
& \left.\quad+4 t_{(1)}^{3}+4 t_{(3)}^{3}+12 t_{(-4)} t_{(-2)} t_{(1)}-12 t_{(-4)} t_{(2)} t_{(3)}-12 t_{(1)} t_{(-1)}-12 t_{(3)} t_{(-3)}\right)
\end{aligned}
$$

\section{Poisson structure of a trinion}

We can now derive the explicit forms of Poisson brackets for Euler characteristic -1 surfaces; that is, surfaces with fundamental groups free of rank 2 . We begin with the three-holed sphere.

Theorem 25. Let $\mathfrak{X}$ be the relative character variety of $S=\Sigma_{3,0}$. Then the Poisson bracket has the following properties which determines all pairings of elements in $\mathbb{C}[\mathfrak{X}]: t_{( \pm 1)}, t_{( \pm 2)}, t_{( \pm 3)}$ are Casimirs,

$$
\begin{aligned}
& \mathfrak{a}_{4,-4}=\left\{t_{(4)}, t_{(-4)}\right\}=P-2 t_{(5)}, \text { and } \\
& \mathfrak{a}_{ \pm 4,5}=\left\{t_{( \pm 4)}, t_{(5)}\right\}=\frac{t_{(5)}\left\{t_{( \pm 4)}, P\right\}-\left\{t_{( \pm 4)}, Q\right\}}{\left\{t_{(-4)}, t_{(4)}\right\}}= \pm \frac{\partial\left(Q-t_{(5)} P\right)}{\partial t_{(\mp 4)}} .
\end{aligned}
$$

For any Poisson bracket there exists an exterior bi-vector field whose restriction to symplectic leaves corresponds to the symplectic form. Denote this bi-vector by $\mathfrak{a}$, and let $f, g \in \mathbb{C}[\mathfrak{X}]$. Then with respect to interior multiplication $\{f, g\}=\mathfrak{a} \cdot d f \otimes d g$. In local coordinates $\left(z_{1}, \ldots, z_{k}\right)$ it takes the form

$$
\mathfrak{a}=\sum_{i, j} \mathfrak{a}_{i, j} \frac{\partial}{\partial z_{i}} \wedge \frac{\partial}{\partial z_{j}}
$$


and so

$$
\begin{aligned}
\{f, g\} & =\sum_{i, j}\left(\mathfrak{a}_{i, j} \frac{\partial}{\partial z_{i}} \wedge \frac{\partial}{\partial z_{j}}\right) \cdot\left(\frac{\partial f}{\partial z_{i}} d z_{i} \otimes \frac{\partial g}{\partial z_{j}} d z_{j}\right) \\
& =\sum_{i, j} \mathfrak{a}_{i, j}\left(\frac{\partial f}{\partial z_{i}} \frac{\partial g}{\partial z_{j}}-\frac{\partial f}{\partial z_{j}} \frac{\partial g}{\partial z_{i}}\right) .
\end{aligned}
$$

Theorem 25. the calculations derived in its proof (see below), and observing the symmetry between $\left\{t_{(4)}, t_{(5)}\right\}$ and $\left\{t_{(-4)}, t_{(5)}\right\}$ allow for a succinct expression of the Poisson bi-vector field and prove the following corollary.

Corollary 26. $\mathfrak{a}_{-4,5}=-\mathfrak{i}\left(\mathfrak{a}_{4,5}\right)$ where $\mathfrak{i}=\mathfrak{i}_{1} \mathfrak{t i}_{1} \mathfrak{t}$ is the outer automorphism $\mathrm{x}_{i} \mapsto$ $\mathrm{x}_{i}^{-1}$, and the Poisson bi-vector field for the relative $\mathrm{SL}(3, \mathbb{C})$-character variety of $\Sigma_{3,0}$ is

$$
\left(P-2 t_{(5)}\right) \frac{\partial}{\partial t_{(4)}} \wedge \frac{\partial}{\partial t_{(-4)}}+(1-\mathfrak{i})\left(\frac{\partial\left(Q-t_{(5)} P\right)}{\partial t_{(-4)}} \frac{\partial}{\partial t_{(4)}} \wedge \frac{\partial}{\partial t_{(5)}}\right) .
$$

Proof of Theorem 25. Since a Poisson bracket is a bilinear, anti-commutative derivation, it is completely determined once it is formulated on the generators of $\mathbb{C}[\mathfrak{X}]$.

We present the fundamental group of $S=\Sigma_{3,0}$ as

$$
\pi_{1}(S)=\left\{\mathrm{x}_{1}, \mathrm{x}_{2}, \mathrm{x}_{3} \mid \mathrm{x}_{3} \mathrm{x}_{2} \mathrm{x}_{1}=1\right\}
$$

so $\mathrm{x}_{3}=\mathrm{x}_{1}^{-1} \mathrm{x}_{2}^{-1}$. Hence $\pi_{1}(S)$ is free of rank 2 .

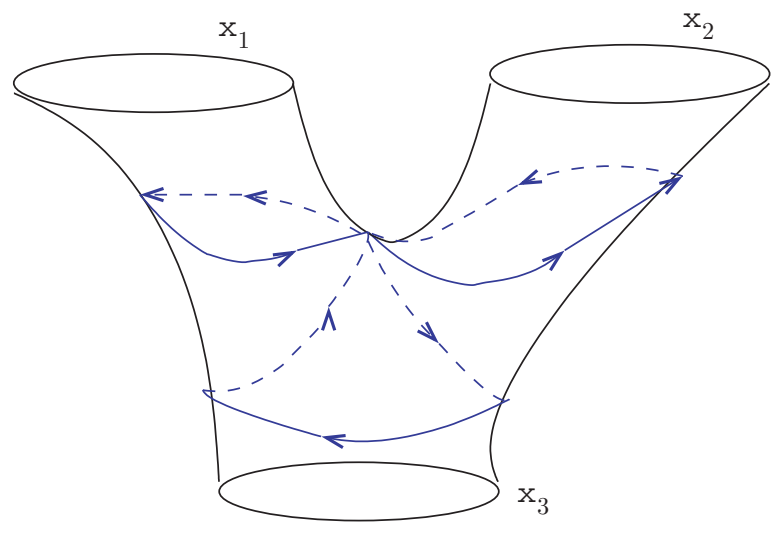

Figure 2. Presentation of $\pi_{1}\left(\Sigma_{3,0}, *\right)$

The boundary curves in $S$ are the words $\mathrm{x}_{1}, \mathrm{x}_{2}$, and $\left(\mathrm{x}_{2} \mathrm{x}_{1}\right)^{-1}$, which are disjoint in the surface. The sum in formula (2) is taken over intersections, and is welldefined on homotopy classes. So the traces of words corresponding to disjoint curves Poisson commute; that is, they are Casimirs. Hence $t_{( \pm i)}$ are Casimirs, for $1 \leq i \leq 3$, since they correspond to traces of boundary curves (and their inverses) in $S$.

Using the derivation property and the identity $t_{(5)}^{2}-P t_{(5)}+Q=0$, we deduce

$$
t_{(5)}\left\{t_{( \pm 4)}, P\right\}+P\left\{t_{( \pm 4)}, t_{(5)}\right\}-\left\{t_{( \pm 4)}, Q\right\}=\left\{t_{( \pm 4)}, t_{(5)}^{2}\right\}=2 t_{(5)}\left\{t_{( \pm 4)}, t_{(5)}\right\} .
$$


Hence

$$
\left(2 t_{(5)}-P\right)\left\{t_{( \pm 4)}, t_{(5)}\right\}=t_{(5)}\left\{t_{( \pm 4)}, P\right\}-\left\{t_{( \pm 4)}, Q\right\} .
$$

So (9) follows from (8).

Now assuming (8) and subsequently using the explicit expressions of $P$ and $Q$ given in Section 5, we further derive explicit expressions for (9) as follows:

$$
\begin{aligned}
\left\{t_{(4)}, P\right\}= & \left(P-2 t_{(5)}\right)\left(t_{(4)}-t_{(1)} t_{(-2)}\right) \\
\left\{t_{(4)}, Q\right\}= & \left(P-2 t_{(5)}\right)\left(-6 t_{(4)}+3 t_{(-4)}^{2}-3 t_{(-1)} t_{(-3)}-3 t_{(2)} t_{(3)}+3 t_{(1)} t_{(-2)}\right. \\
& +t_{(1)} t_{(-1)} t_{(4)}+t_{(2)} t_{(-2)} t_{(4)}+t_{(3)} t_{(-3)} t_{(4)}+t_{(-1)}^{2} t_{(-2)}+t_{(1)}^{2} t_{(-3)} \\
& +t_{(2)} t_{(-3)}^{2}+t_{(1)} t_{(2)}^{2}+t_{(3)} t_{(-2)}^{2}+t_{(-1)} t_{(3)}^{2}+t_{(-1)}^{2} t_{(2)}^{2}-t_{(1)} t_{(-1)} t_{(2)} t_{(3)} \\
& -t_{(-3)} t_{(-2)} t_{(-1)} t_{(2)}-t_{(1)} t_{(2)} t_{(-2)}^{2}-t_{(-2)} t_{(-1)} t_{(1)}^{2}+2 t_{(1)} t_{(3)} t_{(-4)} \\
& \left.+2 t_{(-2)} t_{(-3)} t_{(-4)}-4 t_{(-1)} t_{(2)} t_{(-4)}\right) \\
\left\{t_{(-4)}, P\right\}= & \left(2 t_{(5)}-P\right)\left(t_{(-4)}-t_{(-1)} t_{(2)}\right) \\
\left\{t_{(-4)}, Q\right\}= & \left(2 t_{(5)}-P\right)\left(-6 t_{(-4)}+3 t_{(4)}^{2}-3 t_{(1)} t_{(3)}-3 t_{(-2)} t_{(-3)}+3 t_{(-1)} t_{(2)}\right. \\
& +t_{(1)} t_{(-1)} t_{(-4)}+t_{(2)} t_{(-2)} t_{(-4)}+t_{(3)} t_{(-3)} t_{(-4)}+t_{(1)}^{2} t_{(2)}+t_{(-1)}^{2} t_{(3)} \\
& +t_{(-2)} t_{(3)}^{2}+t_{(-1)} t_{(-2)}^{2}+t_{(-3)} t_{(2)}^{2}+t_{(1)} t_{(-3)}^{2}+t_{(1)}^{2} t_{(-2)}^{2} \\
& -t_{(1)} t_{(-1)} t_{(-2)} t_{(-3)}-t_{(3)} t_{(-2)} t_{(1)} t_{(2)}-t_{(-1)} t_{(-2)} t_{(2)}^{2}-t_{(2)} t_{(1)} t_{(-1)}^{2} \\
& \left.+2 t_{(-1)} t_{(-3)} t_{(4)}+2 t_{(2)} t_{(3)} t_{(4)}-4 t_{(1)} t_{(-2)} t_{(4)}\right)
\end{aligned}
$$

and so

$$
\begin{aligned}
\left\{t_{(4)}, t_{(5)}\right\}= & t_{(4)}\left(t_{(1)} t_{(-1)}+t_{(2)} t_{(-2)}+t_{(3)} t_{(-3)}-t_{(5)}-6\right) \\
& +t_{(-4)}\left(2 t_{(1)} t_{(3)}+2 t_{(-2)} t_{(-3)}-4 t_{(-1)} t_{(2)}\right) \\
& +t_{(5)} t_{(1)} t_{(-2)}+3 t_{(-4)}^{2}-3 t_{(-1)} t_{(-3)}-3 t_{(2)} t_{(3)}+3 t_{(1)} t_{(-2)} \\
& +t_{(-1)}^{2} t_{(-2)}+t_{(1)}^{2} t_{(-3)}+t_{(2)} t_{(-3)}^{2}+t_{(1)} t_{(2)}^{2}+t_{(3)} t_{(-2)}^{2} \\
& +t_{(-1)} t_{(3)}^{2}+t_{(-1)}^{2} t_{(2)}^{2}-t_{(1)} t_{(-1)} t_{(2)} t_{(3)}-t_{(-3)} t_{(-2)} t_{(-1)} t_{(2)} \\
& -t_{(1)} t_{(2)} t_{(-2)}^{2}-t_{(-2)} t_{(-1)} t_{(1)}^{2}, \\
\left\{t_{(-4)}, t_{(5)}\right\}= & t_{(-4)}\left(t_{(5)}-t_{(-1)} t_{(1)}-t_{(2)} t_{(-2)}-t_{(3)} t_{(-3)}+6\right) \\
& +t_{(4)}\left(4 t_{(1)} t_{(-2)}-2 t_{(-1)} t_{(-3)}-2 t_{(2)} t_{(3)}\right) \\
& -t_{(5)} t_{(-1)} t_{(2)}-3 t_{(4)}^{2}+3 t_{(1)} t_{(3)}+3 t_{(-2)} t_{(-3)}-3 t_{(-1)} t_{(2)} \\
& -t_{(1)}^{2} t_{(2)}-t_{(-1)}^{2} t_{(3)}-t_{(-2)} t_{(3)}^{2}-t_{(-1)} t_{(-2)}^{2}-t_{(-3)} t_{(2)}^{2} \\
& -t_{(1)} t_{(-3)}^{2}-t_{(1)}^{2} t_{(-2)}^{2}+t_{(-1)} t_{(1)} t_{(-2)} t_{(-3)}+t_{(3)} t_{(2)} t_{(1)} t_{(-2)} \\
& +t_{(-1)} t_{(-2)} t_{(2)}^{2}+t_{(2)} t_{(1)} t_{(-1)}^{2} .
\end{aligned}
$$

It remains to compute $\left\{t_{(4)}, t_{(-4)}\right\}$. Following the results in G5], we consider immersed closed curves freely homotopic to $\alpha=\mathrm{x}_{1} \mathrm{x}_{2}^{-1}$ and $\beta=\mathrm{x}_{2} \mathrm{x}_{1}^{-1}$ intersecting only at transverse double points.

Since $S$ is homotopic to a closed rectangle with two open disks removed, we depict all curves as in Figure 3 .

We further let $\alpha_{p}$ and $\beta_{p}$ be the curves corresponding to $\alpha$ and $\beta$ based at the point $p$ in $\pi_{1}(S, p)$.

Respectively, let $\alpha_{q}$ and $\beta_{q}$ be the corresponding curves in $\pi_{1}(S, q)$. 


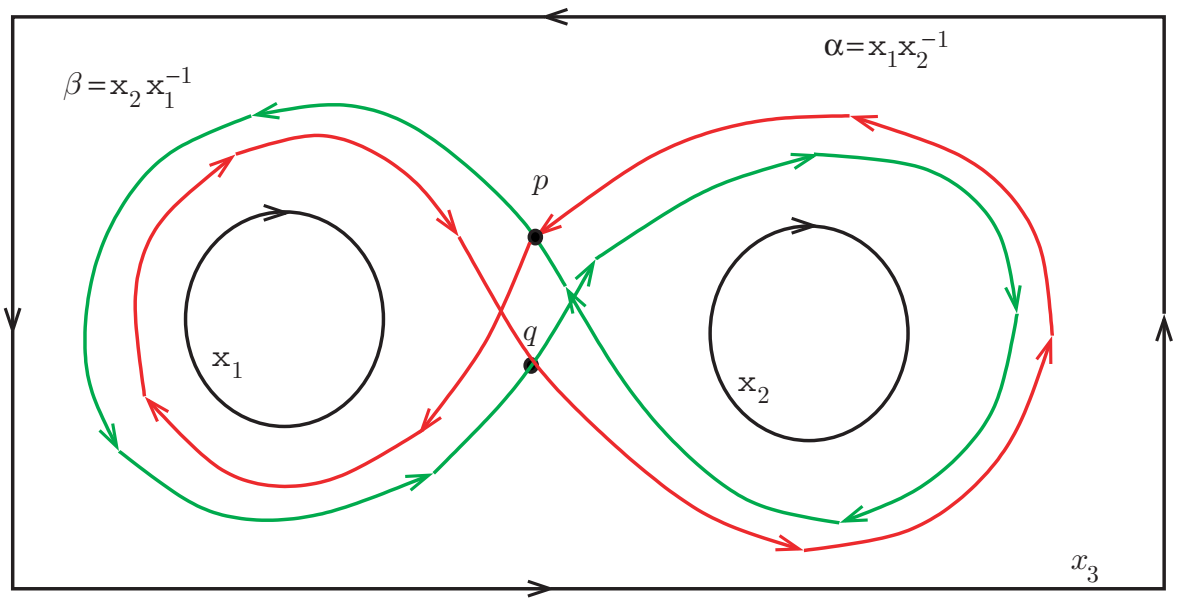

Figure 3. $\alpha$ and $\beta$ in $S$

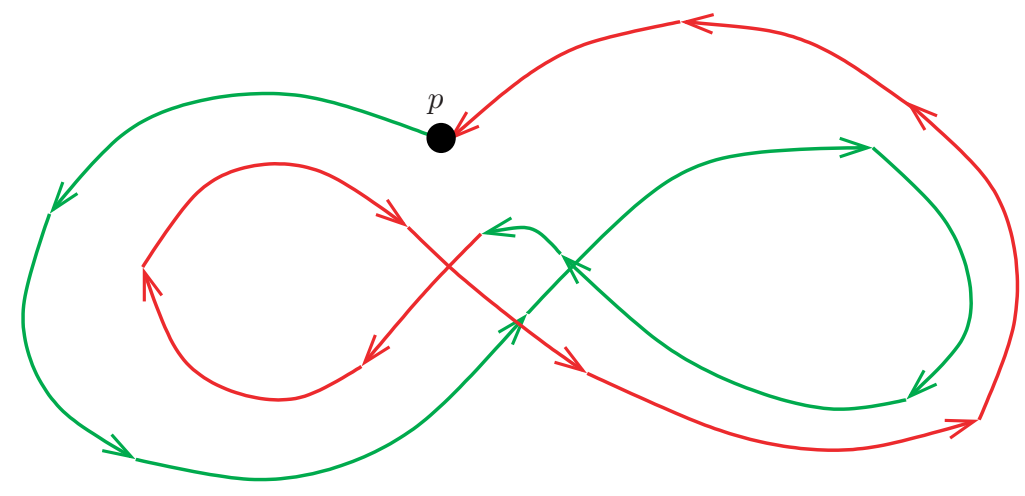

FiguRe 4. $\alpha_{p} \beta_{p}=\mathrm{x}_{2}^{-1} \mathrm{x}_{1} \mathrm{x}_{2} \mathrm{x}_{1}^{-1}$

Calculating the oriented intersection number at $p$ and $q$ we find $\epsilon(p, \alpha, \beta)=-1$ and $\epsilon(q, \alpha, \beta)=1$.

Hence formula (2) and Figures 415, and 6 give

$$
\begin{aligned}
\left\{t_{(4)}, t_{(-4)}\right\}= & \{\operatorname{tr}(\rho(\alpha)), \operatorname{tr}(\rho(\beta))\} \\
= & \epsilon(p, \alpha, \beta)\left(\operatorname{tr}\left(\rho\left(\alpha_{p} \beta_{p}\right)\right)-(1 / 3) \operatorname{tr}(\rho(\alpha)) \operatorname{tr}(\rho(\beta))\right) \\
& +\epsilon(q, \alpha, \beta)\left(\operatorname{tr}\left(\rho\left(\alpha_{q} \beta_{q}\right)\right)-(1 / 3) \operatorname{tr}(\rho(\alpha)) \operatorname{tr}(\rho(\beta))\right) \\
= & -\operatorname{tr}\left(\rho\left(\alpha_{p} \beta_{p}\right)\right)+\operatorname{tr}\left(\rho\left(\alpha_{q} \beta_{q}\right)\right) \\
= & -\operatorname{tr}\left(\mathbf{X}_{2}^{-1} \mathbf{X}_{1} \mathbf{X}_{2} \mathbf{X}_{1}^{-1}\right)+\operatorname{tr}\left(\mathbf{X}_{1} \mathbf{X}_{2}^{-1} \mathbf{X}_{1}^{-1} \mathbf{X}_{2}\right) \\
= & -t_{(5)}+t_{(-5)} \\
= & -t_{(5)}+\left(P-t_{(5)}\right)=P-2 t_{(5)} .
\end{aligned}
$$




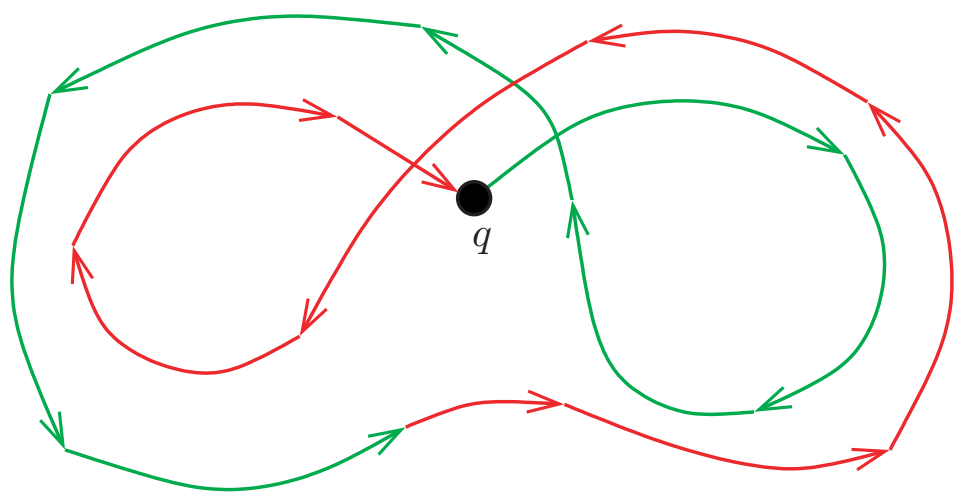

FiguRE 5. $\alpha_{q} \beta_{q}=\mathrm{x}_{1} \mathrm{x}_{2}^{-1} \mathrm{x}_{1}^{-1} \mathrm{x}_{2}$
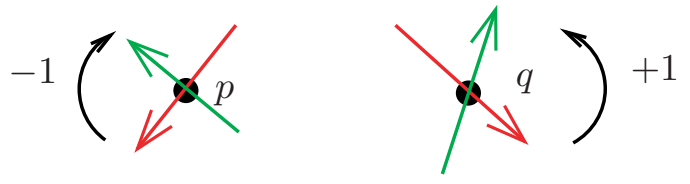

FIGURE 6. Intersection numbers at $p$ and $q$

Comment 27. Formula (9) can be derived in the same manner as we derived formula (8). Doing so leads to the expression

$$
\begin{aligned}
\operatorname{tr}\left(\mathbf{X}_{1} \mathbf{X}_{2}^{-1} \mathbf{X}_{1}^{-1} \mathbf{X}_{2}^{-1} \mathbf{X}_{1} \mathbf{X}_{2}\right) & -\operatorname{tr}\left(\mathbf{X}_{1} \mathbf{X}_{2}^{-1}\right)+\operatorname{tr}\left(\mathbf{X}_{2}^{-2} \mathbf{X}_{1}^{2} \mathbf{X}_{2} \mathbf{X}_{1}^{-1}\right) \\
& -\operatorname{tr}\left(\mathbf{X}_{2}^{-1} \mathbf{X}_{1} \mathbf{X}_{2}^{-1} \mathbf{X}_{1} \mathbf{X}_{2} \mathbf{X}_{1}^{-1}\right)
\end{aligned}
$$

Subsequently using polynomial trace relations to reduce these trace expressions to polynomials in $t_{(i)}$ for $1 \leq|i| \leq 5$ has provided us with further verification of (9).

\section{Poisson structure on a one-holed torus}

The only other orientable surface with Euler characteristic -1 is the one-holed torus; that is, $S^{1} \times S^{1}$ with a disk removed. Let $T=\Sigma_{1,1}$ be the one-holed torus. Its fundamental group is presentable as

$$
\pi_{1}(T)=\left\{\mathrm{x}_{1}, \mathrm{x}_{2}, \mathrm{x}_{3} \mid \mathrm{x}_{1} \mathrm{x}_{2} \mathrm{x}_{1}^{-1} \mathrm{x}_{2}^{-1} \mathrm{x}_{3}=1\right\} .
$$

With respect to this presentation the boundary $\mathrm{x}_{3}$ corresponds to the inverse of the word $\mathrm{x}_{1} \mathrm{x}_{2} \mathrm{x}_{1}^{-1} \mathrm{x}_{2}^{-1}$. Consequently, the only Casimir that is also a generator is $t_{(5)}$ since it corresponds to $\operatorname{tr}\left(\mathbf{X}_{1} \mathbf{X}_{2} \mathbf{X}_{1}^{-1} \mathbf{X}_{2}^{-1}\right)$. Thus the 81 pairings coming from the 9 generators is reduced to 64 . Anticommutativity reduces this number to 28 .

Observe that $\left\{t_{(i)}, t_{(-i)}\right\}=0$ since the corresponding curves are homotopic in $T$ to parallel curves and so have no intersection. Note that this is not always true in general; in the case of the three-holed sphere $\left\{t_{(4)}, t_{(-4)}\right\} \neq 0$. We are left with 24 computations. 


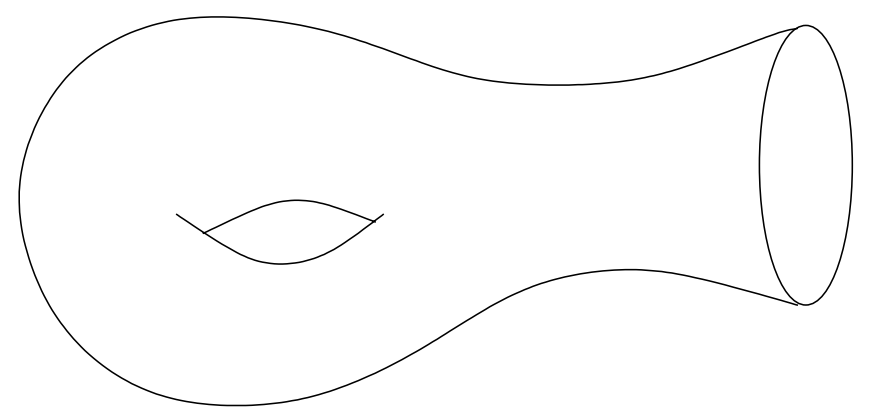

Figure 7. One-Holed Torus

We first address the pairings which come from cycles of word length one and so only have one intersection.

Let $\alpha$ be homotopic to $\mathrm{x}_{1}$ and $\beta$ be homotopic to $\mathrm{x}_{2}$, as in Figure 8 ,

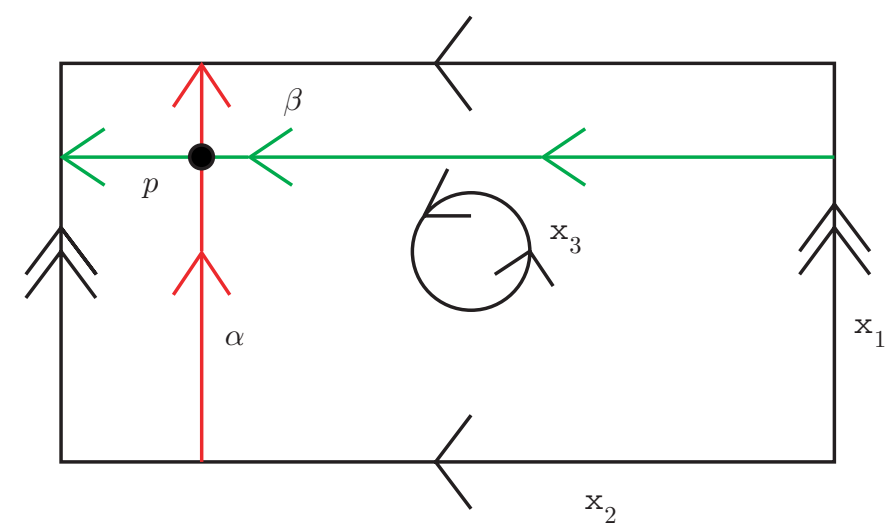

Figure 8. $\alpha=\mathrm{x}_{1}$ and $\beta=\mathrm{x}_{2}$ in $T$

Let $\epsilon(p, \alpha, \beta)=\epsilon_{p}$ to simplify the notation.

Then

$$
\begin{aligned}
\left\{t_{(1)}, t_{(2)}\right\} & =\epsilon_{p}\left(\operatorname{tr}\left(\rho\left(\alpha_{p} \beta_{p}\right)\right)-(1 / 3) \operatorname{tr}(\rho(\alpha)) \operatorname{tr}(\rho(\beta))\right) \\
& =\epsilon_{p}\left(\operatorname{tr}\left(\mathbf{X}_{1} \mathbf{X}_{2}\right)-(1 / 3) \operatorname{tr}\left(\mathbf{X}_{1}\right) \operatorname{tr}\left(\mathbf{X}_{2}\right)\right) \\
& =\operatorname{tr}\left(\mathbf{X}_{1} \mathbf{X}_{2}\right)-(1 / 3) \operatorname{tr}\left(\mathbf{X}_{1}\right) \operatorname{tr}\left(\mathbf{X}_{2}\right) \\
& =t_{(3)}-(1 / 3) t_{(1)} t_{(2)}
\end{aligned}
$$

Likewise, we can compute

$$
\begin{aligned}
\left\{t_{(-1)}, t_{(2)}\right\} & =-t_{(-4)}+(1 / 3) t_{(-1)} t_{(2)}, \\
\left\{t_{(1)}, t_{(-2)}\right\} & =-t_{(4)}+(1 / 3) t_{(1)} t_{(-2)}, \\
\left\{t_{(-1)}, t_{(-2)}\right\} & =t_{(-3)}-(1 / 3) t_{(-1)} t_{(-2)} .
\end{aligned}
$$


We can already see symmetry coming from $\operatorname{Out}\left(\mathrm{F}_{2}\right)$. In $\mathfrak{D} \cong D_{4}$ define $\mathfrak{i}_{2}=\mathfrak{t i}_{1} \mathfrak{t}$, the mapping which sends $\mathrm{x}_{2} \mapsto \mathrm{x}_{2}^{-1}$. Then

$$
\begin{aligned}
\left\{t_{(-1)}, t_{(2)}\right\} & =-\mathfrak{i}_{1}\left\{t_{(1)}, t_{(2)}\right\}=\left\{\mathfrak{i}_{1} t_{(1)}, \mathfrak{i}_{1} t_{(2)}\right\}, \\
\left\{t_{(1)}, t_{(-2)}\right\} & =-\mathfrak{i}_{2}\left\{t_{(1)}, t_{(2)}\right\}=\left\{\mathfrak{i}_{2} t_{(1)}, \mathfrak{i}_{2} t_{(2)}\right\}, \\
\left\{t_{(-1)}, t_{(-2)}\right\} & =\mathfrak{i}\left\{t_{(1)}, t_{(2)}\right\}=\left\{\mathfrak{i} t_{(1)}, \mathfrak{i} t_{(2)}\right\} .
\end{aligned}
$$

We are left with 20 computations.

Next we consider curves which again intersect only once, but at least one of them has word length two. There will be two cases: either you have a repeated letter or you get a cancellation after cyclic reduction. We demonstrate both cases here and then state the other pairings of these types. First we consider the case of the paired cycles having a common letter and where no letter between them cancels.

For instance, let $\alpha=\mathrm{x}_{1}$ and $\beta=\mathrm{x}_{1} \mathrm{x}_{2}$ (both have the letter $\mathrm{x}_{1}$, and when concatenated the word is cyclically reduced).

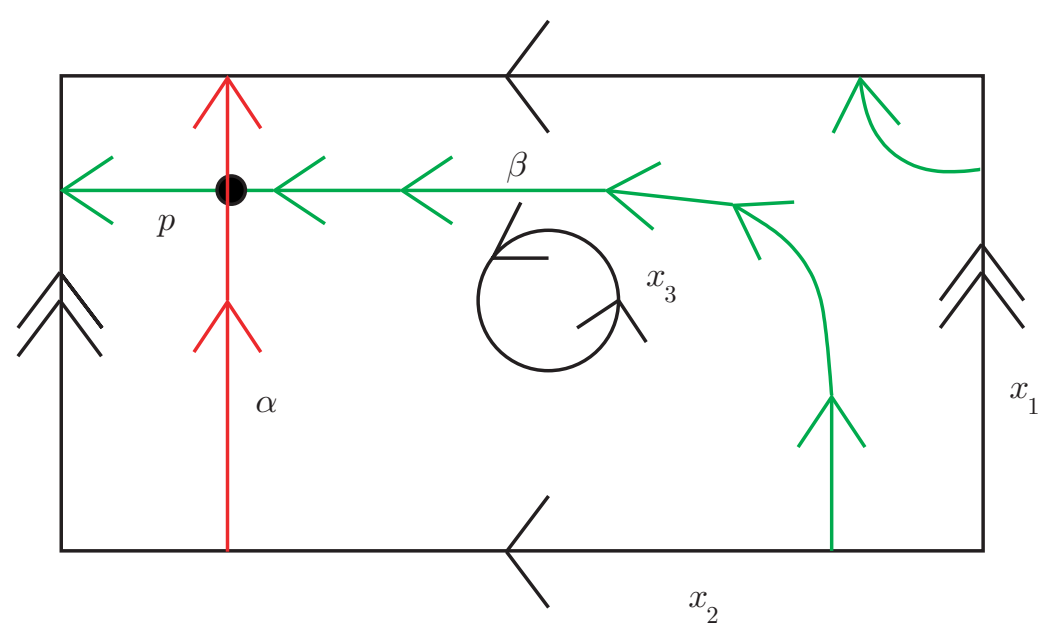

Figure 9. $\alpha=\mathrm{x}_{1}$ and $\beta=\mathrm{x}_{1} \mathrm{x}_{2}$ in $T$

Then

$$
\begin{aligned}
\left\{t_{(1)}, t_{(3)}\right\} & =\epsilon_{p}\left(\operatorname{tr}\left(\rho\left(\alpha_{p} \beta_{p}\right)\right)-(1 / 3) \operatorname{tr}(\rho(\alpha)) \operatorname{tr}(\rho(\beta))\right) \\
& =\operatorname{tr}\left(\mathbf{X}_{1}^{2} \mathbf{X}_{2}\right)-(1 / 3) \operatorname{tr}\left(\mathbf{X}_{1}\right) \operatorname{tr}\left(\mathbf{X}_{1} \mathbf{X}_{2}\right) \\
& =-t_{(-1)} t_{(2)}+t_{(-4)}+(2 / 3) t_{(1)} t_{(3)} .
\end{aligned}
$$

There are seven more cycles corresponding to minimal generators having a common letter and one intersection. They all are computed like the one above. Again 
there is apparent symmetry coming from $\mathfrak{D}$.

$$
\begin{aligned}
& \left\{t_{(-1)}, t_{(-3)}\right\}=-t_{(1)} t_{(-2)}+t_{(4)}+(2 / 3) t_{(-1)} t_{(-3)}=\mathfrak{i}\left\{t_{(1)}, t_{(3)}\right\}=\left\{\mathfrak{i} t_{(1)}, \mathfrak{i} t_{(3)}\right\}, \\
& \left\{t_{(1)}, t_{(4)}\right\}=t_{(-1)} t_{(-2)}-t_{(-3)}-(2 / 3) t_{(1)} t_{(4)}=-\mathfrak{i}_{2}\left\{t_{(1)}, t_{(3)}\right\}=\left\{\mathfrak{i}_{2} t_{(1)}, \mathfrak{i}_{2} t_{(3)}\right\}, \\
& \left\{t_{(-1)}, t_{(-4)}\right\}=t_{(1)} t_{(2)}-t_{(3)}-(2 / 3) t_{(-1)} t_{(-4)}=-\mathfrak{i}_{1}\left\{t_{(1)}, t_{(3)}\right\}=\left\{\mathfrak{i}_{1} t_{(1)}, \mathfrak{i}_{1} t_{(3)}\right\}, \\
& \left\{t_{(2)}, t_{(3)}\right\}=t_{(-2)} t_{(1)}-t_{(4)}-(2 / 3) t_{(2)} t_{(3)}=-\mathfrak{t}\left\{t_{(1)}, t_{(3)}\right\}=\left\{\mathfrak{t} t_{(1)}, \mathfrak{t} t_{(3)}\right\}, \\
& \left\{t_{(-2)}, t_{(-3)}\right\}=t_{(2)} t_{(-1)}-t_{(-4)}-(2 / 3) t_{(-2)} t_{(-3)}=-\mathfrak{i t}\left\{t_{(1)}, t_{(3)}\right\}=\left\{\mathfrak{i t t} t_{(1)}, \mathfrak{i t t} t_{(3)}\right\}, \\
& \left\{t_{(2)}, t_{(-4)}\right\}=-t_{(-2)} t_{(-1)}+t_{(-3)}+(2 / 3) t_{(2)} t_{(-4)}=\mathfrak{i}_{1} \mathfrak{t}\left\{t_{(1)}, t_{(3)}\right\}=\left\{\mathfrak{i}_{1} \mathfrak{t} t_{(1)}, \mathfrak{i}_{1} \mathfrak{t} t_{(3)}\right\}, \\
& \left\{t_{(-2)}, t_{(4)}\right\}=-t_{(2)} t_{(1)}+t_{(3)}+(2 / 3) t_{(-2)} t_{(4)}=\mathfrak{i}_{2} \mathfrak{t}\left\{t_{(1)}, t_{(3)}\right\}=\left\{\mathfrak{i}_{2} \mathfrak{t} t_{(1)}, \mathfrak{i}_{2} \mathfrak{t} t_{(3)}\right\} \text {. }
\end{aligned}
$$

We are now left with 12 computations.

Now we consider the other case of only one intersection in the pair of cycles, where after concatenating there is some non-trivial (reduced word length) cyclic reduction in the word. For instance, let $\alpha=\mathrm{x}_{1}$ and $\beta=\mathrm{x}_{1}^{-1} \mathrm{x}_{2}^{-1}$.

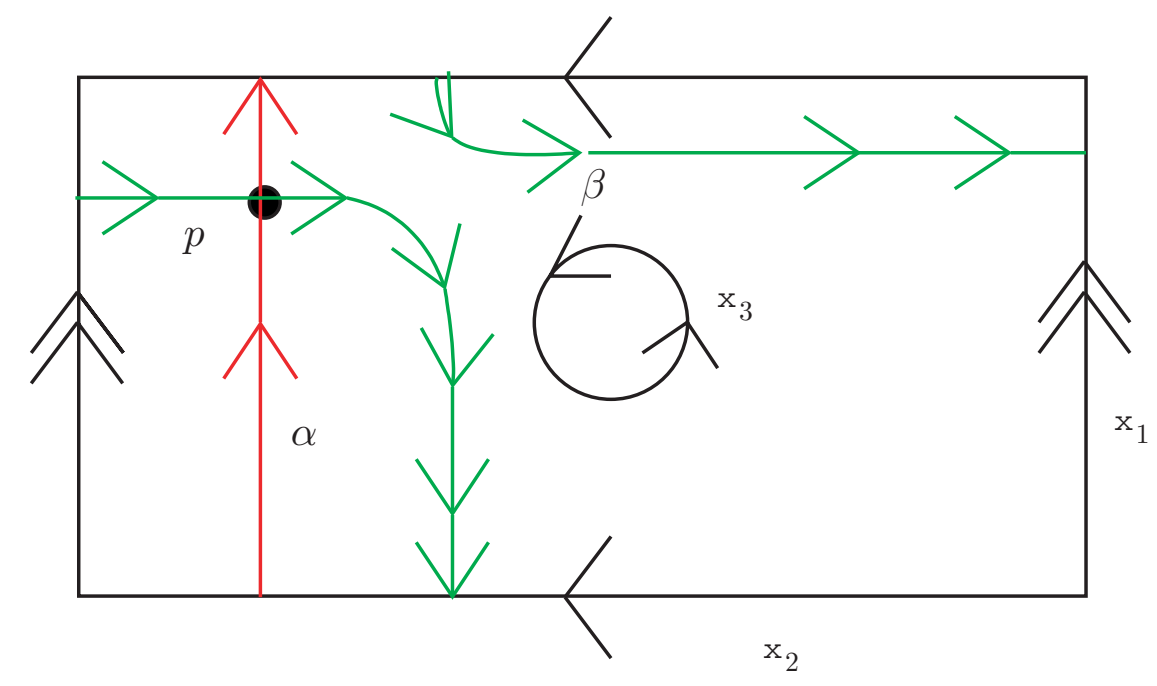

FiguRE 10. $\alpha=\mathrm{x}_{1}$ and $\beta=\mathrm{x}_{1}^{-1} \mathrm{x}_{2}^{-1}$ in $T$

Then

$$
\begin{aligned}
\left\{t_{(1)}, t_{(-3)}\right\} & =\epsilon_{p}\left(\operatorname{tr}\left(\rho\left(\alpha_{p} \beta_{p}\right)\right)-(1 / 3) \operatorname{tr}(\rho(\alpha)) \operatorname{tr}(\rho(\beta))\right) \\
& =(-1)\left(\operatorname{tr}\left(\mathbf{X}_{1} \mathbf{X}_{2}^{-1} \mathbf{X}_{1}^{-1}\right)-(1 / 3) \operatorname{tr}\left(\mathbf{X}_{1}\right) \operatorname{tr}\left(\mathbf{X}_{1}^{-1} \mathbf{X}_{2}^{-1}\right)\right) \\
& =-t_{(-2)}+(1 / 3) t_{(1)} t_{(-3)} .
\end{aligned}
$$

There are seven more cycles corresponding to minimal generators having a canceled letter and one intersection. They all are computed like the one above. Again there is apparent symmetry coming from $\mathfrak{D}$. 


$$
\begin{aligned}
\left\{t_{(-1)}, t_{(3)}\right\} & =-t_{(2)}+(1 / 3) t_{(-1)} t_{(3)}=\mathfrak{i}\left\{t_{(1)}, t_{(-3)}\right\}=\left\{\mathfrak{i} t_{(1)}, \mathfrak{i} t_{(-3)}\right\}, \\
\left\{t_{(1)}, t_{(-4)}\right\} & =t_{(2)}-(1 / 3) t_{(1)} t_{(-4)}=-\mathfrak{i}_{2}\left\{t_{(1)}, t_{(-3)}\right\}=\left\{\mathfrak{i}_{2} t_{(1)}, \mathfrak{i}_{2} t_{(-3)}\right\}, \\
\left\{t_{(-1)}, t_{(4)}\right\} & =t_{(-2)}-(1 / 3) t_{(-1)} t_{(4)}=-\mathfrak{i}_{1}\left\{t_{(1)}, t_{(-3)}\right\}=\left\{\mathfrak{i}_{1} t_{(1)}, \mathfrak{i}_{1} t_{(-3)}\right\}, \\
\left\{t_{(2)}, t_{(-3)}\right\} & =t_{(-1)}-(1 / 3) t_{(2)} t_{(-3)}=-\mathfrak{t}\left\{t_{(1)}, t_{(-3)}\right\}=\left\{\mathfrak{t} t_{(1)}, \mathfrak{t} t_{(-3)}\right\}, \\
\left\{t_{(-2)}, t_{(3)}\right\} & =t_{(1)}-(1 / 3) t_{(-2)} t_{(3)}=-\mathfrak{i t}\left\{t_{(1)}, t_{(-3)}\right\}=\left\{\mathfrak{i t} t_{(1)}, \mathfrak{i t} t_{(-3)}\right\}, \\
\left\{t_{(2)}, t_{(4)}\right\} & =-t_{(1)}+(1 / 3) t_{(2)} t_{(4)}=\mathfrak{i}_{1} \mathfrak{t}\left\{t_{(1)}, t_{(-3)}\right\}=\left\{\mathfrak{i}_{1} \mathfrak{t} t_{(1)}, \mathfrak{i}_{1} \mathfrak{t} t_{(-3)}\right\}, \\
\left\{t_{(-2)}, t_{(-4)}\right\} & =-t_{(-1)}+(1 / 3) t_{(-2)} t_{(-4)}=\mathfrak{i}_{2} \mathfrak{t}\left\{t_{(1)}, t_{(-3)}\right\}=\left\{\mathfrak{i}_{2} \mathfrak{t} t_{(1)}, \mathfrak{i}_{2} \mathfrak{t} t_{(-3)}\right\} .
\end{aligned}
$$

We are now left with 4 computations.

The last case to consider is when there are two intersections. For instance, let $\alpha=\mathrm{x}_{1} \mathrm{x}_{2}$ and $\beta=\mathrm{x}_{1} \mathrm{x}_{2}^{-1}$.

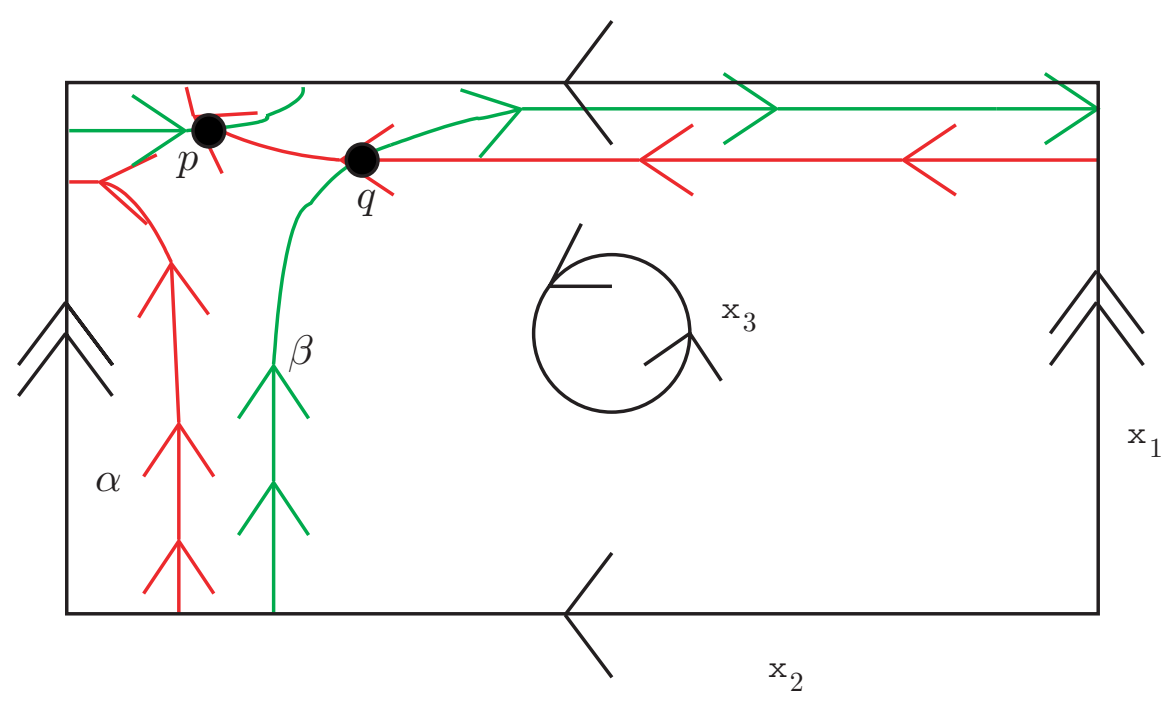

Figure 11. $\alpha=\mathrm{x}_{1} \mathrm{x}_{2}$ and $\beta=\mathrm{x}_{1} \mathrm{x}_{2}^{-1}$ in $T$

Then

$$
\begin{aligned}
\left\{t_{(3)}, t_{(4)}\right\} & =\epsilon_{p}\left(\operatorname{tr}\left(\rho\left(\alpha_{p} \beta_{p}\right)\right)-(1 / 3) \operatorname{tr}(\rho(\alpha)) \operatorname{tr}(\rho(\beta))\right) \\
& +\epsilon_{q}\left(\operatorname{tr}\left(\rho\left(\alpha_{q} \beta_{q}\right)\right)-(1 / 3) \operatorname{tr}(\rho(\alpha)) \operatorname{tr}(\rho(\beta))\right) \\
& =(-1)\left(\operatorname{tr}\left(\mathbf{X}_{2} \mathbf{X}_{1} \mathbf{X}_{2}^{-1} \mathbf{X}_{1}\right)-(1 / 3) \operatorname{tr}\left(\mathbf{X}_{1} \mathbf{X}_{2}\right) \operatorname{tr}\left(\mathbf{X}_{1} \mathbf{X}_{2}^{-1}\right)\right) \\
& +(-1)\left(\operatorname{tr}\left(\mathbf{X}_{2} \mathbf{X}_{1} \mathbf{X}_{1} \mathbf{X}_{2}^{-1}\right)-(1 / 3) \operatorname{tr}\left(\mathbf{X}_{1} \mathbf{X}_{2}\right) \operatorname{tr}\left(\mathbf{X}_{1} \mathbf{X}_{2}^{-1}\right)\right) \\
& =-\operatorname{tr}\left(\mathbf{X}_{1}^{2}\right)-\operatorname{tr}\left(\mathbf{X}_{1} \mathbf{X}_{2} \mathbf{X}_{1} \mathbf{X}_{2}^{-1}\right)+(2 / 3) \operatorname{tr}\left(\mathbf{X}_{1} \mathbf{X}_{2}\right) \operatorname{tr}\left(\mathbf{X}_{1} \mathbf{X}_{2}^{-1}\right) \\
& =-t_{(1)}^{2}+t_{(-1)}-t_{(-4)} t_{(-2)}-t_{(2)} t_{(-3)}+t_{(-1)} t_{(2)} t_{(-2)}-\frac{1}{3} t_{(3)} t_{(4)} .
\end{aligned}
$$

The last identity is a consequence of trace reduction formulas found in $[\mathrm{L}$. 
In a like manner one can compute the follow other brackets of this type:

$$
\begin{aligned}
\left\{t_{(-3)}, t_{(-4)}\right\} & =-t_{(-1)}^{2}+t_{(1)}-t_{(4)} t_{(2)}-t_{(-2)} t_{(3)}+t_{(1)} t_{(-2)} t_{(2)}-\frac{1}{3} t_{(-3)} t_{(-4)} \\
& =\mathfrak{i}\left\{t_{(3)}, t_{(4)}\right\}=\left\{\mathfrak{i} t_{(3)}, \mathfrak{i t} t_{(4)}\right\}, \\
\left\{t_{(3)}, t_{(-4)}\right\} & =t_{(2)}^{2}-t_{(-2)}+t_{(4)} t_{(-1)}+t_{(1)} t_{(-3)}-t_{(-2)} t_{(1)} t_{(-1)}+\frac{1}{3} t_{(3)} t_{(-4)} \\
& =-\mathfrak{t}\left\{t_{(3)}, t_{(4)}\right\}=\left\{\mathfrak{t} t_{(3)}, \mathfrak{t} t_{(4)}\right\} \\
\left\{t_{(-3)}, t_{(4)}\right\} & =t_{(-2)}^{2}-t_{(2)}+t_{(-4)} t_{(1)}+t_{(-1)} t_{(3)}-t_{(2)} t_{(-1)} t_{(1)}+\frac{1}{3} t_{(-3)} t_{(4)} \\
& =-\mathfrak{i t}\left\{t_{(3)}, t_{(4)}\right\}=\left\{\mathfrak{i t} t_{(3)}, \mathfrak{i t} t_{(4)}\right\} .
\end{aligned}
$$

This finishes the bracket computations for the one-holed torus. Observe that the symmetry operators consistently respect the Poisson bracket up to sign in the same way for all minimal generators. In other words, given any element $\mathfrak{d} \in \mathfrak{D}$,

$$
\mathfrak{d}\left(\left\{t_{(i)}, t_{(j)}\right\}\right)= \pm\left\{\mathfrak{d}\left(t_{(i)}\right), \mathfrak{d}\left(t_{(j)}\right)\right\},
$$

for all $i, j$. This compounded with the fact that the bracket is an anti-commutative derivation establishes:

Corollary 28. $\mathfrak{D}$ consists of Poisson homomorphisms and Poisson anti-homomorphisms.

Define the following elements of the group ring of $\mathfrak{D} \cong D_{4}$ acting on $\mathbb{C}[\mathfrak{X}]$ :

- $\Sigma_{1}=1+\mathfrak{i}-\mathfrak{i}_{1}-\mathfrak{i}_{2}$,

- $\Sigma_{2}=1+\mathfrak{i}-\mathfrak{t}-\mathfrak{i t}$

and let $\mathfrak{a}_{i, j}=\left\{t_{(i)}, t_{(j)}\right\}$.

Note

$$
\frac{1}{2} \Sigma_{1} \Sigma_{2}=1+\mathfrak{i}-\mathfrak{i}_{1}-\mathfrak{i}_{2}-\mathfrak{t}-\mathfrak{i t}+\mathfrak{i}_{1} \mathfrak{t}+\mathfrak{i}_{2} \mathfrak{t} .
$$

Then putting our work together from this section proves:

Theorem 29. The Poisson bi-vector field for the $\mathrm{SL}(3, \mathbb{C})$-relative character variety of the one-holed torus is

$$
\begin{aligned}
& \Sigma_{1}\left(\mathfrak{a}_{1,2} \frac{\partial}{\partial t_{(1)}} \wedge \frac{\partial}{\partial t_{(2)}}\right)+\Sigma_{2}\left(\mathfrak{a}_{3,4} \frac{\partial}{\partial t_{(3)}} \wedge \frac{\partial}{\partial t_{(4)}}\right) \\
& +\frac{1}{2} \Sigma_{1} \Sigma_{2}\left(\mathfrak{a}_{1,3} \frac{\partial}{\partial t_{(1)}} \wedge \frac{\partial}{\partial t_{(3)}}+\mathfrak{a}_{1,-3} \frac{\partial}{\partial t_{(1)}} \wedge \frac{\partial}{\partial t_{(-3)}}\right),
\end{aligned}
$$

where:

- $\mathfrak{a}_{1,2}=t_{(3)}-\frac{1}{3} t_{(1)} t_{(2)}$,

- $\mathfrak{a}_{1,3}=\frac{2}{3} t_{(1)} t_{(3)}-t_{(-1)} t_{(2)}+t_{(-4)}$,

- $\mathfrak{a}_{1,-3}=-t_{(-2)}+\frac{1}{3} t_{(1)} t_{(-3)}$,

- $\mathfrak{a}_{3,4}=-t_{(1)}^{2}+t_{(-1)}-t_{(-4)} t_{(-2)}-t_{(2)} t_{(-3)}+t_{(-1)} t_{(2)} t_{(-2)}-\frac{1}{3} t_{(3)} t_{(4)}$.

Observe that $\mathfrak{a}_{1,3}=\mathfrak{n}_{(2)}\left(\mathfrak{a}_{1,2}\right)$ and $\mathfrak{a}_{1,-3}=-\mathfrak{n}_{(-2)}\left(\mathfrak{a}_{1,2}\right)$, where $\mathfrak{n}_{(2)}$ is the outer automorphism that sends $\mathrm{x}_{1} \mapsto \mathrm{x}_{1}$ and $\mathrm{x}_{2} \mapsto \mathrm{x}_{1} \mathrm{x}_{2}$ and where $\mathfrak{n}_{(-2)}$ is the outer au- 
tomorphism that sends $\mathrm{x}_{1} \mapsto \mathrm{x}_{1}$ and $\mathrm{x}_{2} \mapsto \mathrm{x}_{1}^{-1} \mathrm{x}_{2}^{-1}$. So up to outer automorphisms the bracket is determined by $\mathfrak{a}_{1,2}$ and $\mathfrak{a}_{3,4}$ alone.

Comment 30. Since the boundary of the one-holed torus is interior to its 2-cell and corresponds to a boundary on the exterior of the 2-cell of the three-holed sphere, the orientations are reversed with respect to each other in the above computations. We describe how these two structures relate to each other in an upcoming paper and how this observation is important to understanding a sign difference between the two structures.

\section{ACKNOWLEDGMENTS}

The author thanks his advisor Bill Goldman for introducing him to this topic and for his continuous encouragement and support. This work was completed at Park City Mathematics Institute in 2006. The author thanks PCMI for its financial support and for providing a stimulating environment in which to work. Additionally, he thanks Hans Boden, Carlos Florentino, and Elisha Peterson for fruitful conversations. Lastly, the author thanks the referee whose comments helped make this paper more readable and well organized.

\section{REFERENCES}

[AP] Abeasis, A., and Pittaluga, M., On a minimal set of generators for the invariants of $3 \times 3$ matrices, Comm. Alg. 17(2) (1989), 487-499. MR978487 (90d:15021)

[ADS] Aslaksen, H., Drensky, V., and Sadikova, L., Defining relations of invariants of two $3 \times 3$ matrices, arXiv:math.RA/0405389 v1, 2004. MR2155550(2006b:16034)

[A] Artin, M., On Azumaya algebras and finite dimensional representations of rings, J. of Alg. 11 (1969), 532-563. MR0242890 (39:4217)

[B] Brown, K., "Cohomology of groups," Graduate Texts in Mathematics No. 87, SpringerVerlag, New York, 1982. MR672956 (83k:20002)

[Br] Bredon, G., "Topology and Geometry" Graduate Texts in Mathematics No. 139, Springer-Verlag, New York, 1993. MR.1224675 (94d:55001)

[CLO] Cox, D., Little, J., and O'Shea, D., "Using Algebraic Geometry," Graduate Texts in Mathematics No. 185, Springer-Verlag, New York, 1998. MR1639811 (99h:13033)

[CS] Chas, M., and Sullivan, D., String Topology, arXiv.org/math.GT/9911159, 1999.

[CSM] Carter, R., Segal, G., and Maconald, I., "Lectures on Lie Groups and Lie Algebras," London Mathematical Society Student Texts No. 32, Cambridge University Press, Cambridge, 1995. MR1356712 (97f:17002)

[D] Dolgachev, I., "Lectures on Invariant Theory," London Mathematical Lecture Notes Series 296, Cambridge University Press, 2003. MR2004511 (2004g:14051)

[DF] Drensky, V., and Formanek, E., "Polynomial Identity Rings," Advanced Courses in Mathematics. CRM Barcelona, Birkhäuser Verlag. Basel, 2004. MR2064082 (2005c:16040)

[Du] Dubnov, J., Sur une généralisation de l'équation de Hamilton-Cayley et sur les invariants simultanés de plusieurs affineurs, Proc. Seminar on Vector and Tensor Analysis, Mechanics Research Inst., Moscow State Univ. 2/3 (1935), 351-367.

[DZ] Dufour, J., and Zung, N.T., "Poisson Structures and their Normal Forms," Progress in Mathematics, Vol. 242, Birkhäuser Verlag, Berlin, 2005. MR2178041 (2007b:53170)

[E] Eisenbud, D., "Commutative Algebra with a View Toward Algebraic Geometry," Graduate Texts in Mathematics No. 150, Springer-Verlag, New York, 1995. MR1322960 (97a:13001)

[Fo] Foth, Philip A., Geometry of moduli spaces of flat bundles on punctured surfaces, Internat. J. Math. 9 (1998), no. 1, 63-73. MR1612318 (99i:14014)

[F] Fox, R., Free differential calculus. I, Ann. of Math. 57 (1953), 547-560. MR0053938 (14:843d) 
[G1] Goldman, W., Geometric structures on manifolds and varieties of representations, Contemp. Math. 74 (1988), 169-197. MR957518 (90i:57024)

[G2] Goldman, W., Convex real projective structures on compact surfaces, J. Diff. Geo. 31 (1990), 791-845. MR1053346 (91b:57001)

[G3] Goldman, W., Introduction to Character Varieties, unpublished notes, 2003.

[G4] Goldman, W., The Symplectic Nature of Fundamental Groups of Surfaces, Advances in Math. 54 (1984), 200-225. MR762512 (86i:32042)

[G5] Goldman, W., Invariant functions on Lie groups and Hamiltonian flows of surface group representations, Invent. Math. 85 (1986), 263-302. MR846929 (87j:32069)

[G6] Goldman, W., Mapping class group dynamics on surface group representations in "Problems on Mapping Class Groups and Related Topics," ed. by B. Farb, Proc. Symp. Pure and Applied Math., Vol. 74 (2006), 198-225. MR2264541 (2007h:57020)

[GHJW] Guruprasad, K., Huebschmann, J., Jeffrey, L., and Weinstein, A., Group systems, groupoids, and moduli spaces of parabolic bundles, Duke Math. J. 89 (1997), no. 2, 377-412. MR1460627 (98e:58034)

[GM] Goldman, W., and Millson, J., The deformation theory of representations of fundamental groups of compact Kähler manifolds, Inst. Hautes Études Sci. Publ. Math. No. 67 (1988), 43-96. MR 972343 (90b:32041)

[Ha] Hartshorne, Robin, "Algebraic geometry," Graduate Texts in Mathematics, No. 52. Springer-Verlag, New York-Heidelberg, 1977. xvi+496 pp. MR0463157 (57:3116)

[H] Hungerford, T., "Algebra," Graduate Texts in Mathematics No. 73, Springer-Verlag, New York, 1974. MR600654 (82a:00006)

[Ka] Karshon, Y., An algebraic proof for the symplectic structure of moduli space, Proc. Amer. Math. Soc. 116 (1992), 591-605. MR.1112494 (93a:14010)

[Ki] Kim, H., The symplectic global coordinates on the moduli space of real projective structures, J. Diff. Geo. 53 (1999), 359-401. MR1802726 (2002f:53144)

[Ko] Kostov, Vladimir Petrov, The connectedness of some varieties and the Deligne-Simpson problem, J. Dyn. Control Syst. 11 (2005), no. 1, 125-155. MR2122469 (2006c:15029)

[L] Lawton, S., Generators, Relations and symmetries in pairs of $3 \times 3$ unimodular matrices, J. Algebra 313 (2007), 782-801. MR2329569

[LP] Lawton, S., and Peterson, E., Spin Networks and SL(2, C)-Character Varieties, to appear in the "Handbook of Teichmüller Theory" (A. Papadopoulos, editor), Volume II, EMS Publishing House, Zürich, 2008 (in press).

[MS] Marincuk, A., and Sibirskii, K., Minimal polynomial bases of affine invariants of square matrices of order three, Mat. Issled. 6 (1971), 100-113. MR0289548 (44:6736)

[MKS] Magnus, W., Karrass, A., and Solitar, D., "Combinatorial Group Theory: Presentations of Groups in Terms of Generators and Relations," Pure and Applied Mathematics Vol. XIII, Interscience Publishers, 1966. MR0207802 (34:7617)

[N] Nakamoto, K., The structure of the invariant ring of two matrices of degree 3, J. Pure and Applied Alg. 166 (2002), 125-148. MR:1868542 (2002h:16038)

[P1] Procesi, C., Invariant theory of $n \times n$ matrices, Advances in Mathematics 19 (1976), 306-381. MR0419491 (54:7512)

[P2] Procesi, C., Finite dimensional representations of algebras, Israel J. Math. 19 (1974), 169-182. MR0357507 (50:9975)

[R] Razmyslov, Y., Trace identities of full matrix algebras over a field of characteristic zero. (Russian) Izv. Akad. Nauk SSSR Ser. Mat. 38 (1974), 723-756. MR0506414 (58:22158)

[S] Shafarevich, I., "Basic Algebraic Geometry 1," $2^{\text {nd }}$ edition, Springer-Verlag, Berlin, 1994. MR1328833 (95m:14001)

[Si] Sikora, A., $S L_{n}$-character varieties as space of graphs, Trans. Amer. Math. Soc. 353 (2001), no. 7, 2773-2804. MR.1828473 (2003b:57004)

[SR] Spencer, A., and Rivlin, R., Further results in the theory of matrix polynomials, Arch. Rational Mech. Anal. 4 (1960), 214-230. MR0109830(22:715)

[T] Teranishi, Y., The ring of invariants of matrices, Nagoya Math. J. 104 (1986), 149-161. MR868442 (88a:16038)

[Wa] Warner, Frank W., "Foundations of differentiable manifolds and Lie groups," Graduate Texts in Mathematics No. 94. Springer-Verlag, New York-Berlin, 1983. ix+272. MR722297 (84k:58001) 
[W] Wolpert, S., On the symplectic geometry of deformations of a hyperbolic surface, Ann. of Math. (2) 117 (1983), 207-234. MR690844(85e:32028)

Department of Mathematics, Instituto Superior Técnico, Lisbon, Portugal

E-mail address: slawton@math.ist.utl.pt

$U R L:$ http://www.math.ist.utl.pt/ slawton

Current address: Department of Mathematics, University of Maryland, College Park, Maryland 20742 